\title{
Quorum Sensing and Iron-Dependent Coordinated Control of Autoinducer-2 Production via Small RNA RyhB in Vibrio vulnificus
}

Keun-Woo Lee

Sogang University

Yancheng Wen

Sogang University

Na-Young Park

Sogang University

Kun-Soo Kim ( $\sim$ kskim@sogang.ac.kr)

Sogang University

\section{Research Article}

Keywords: Vibrio vulnificus, Quorum-sensing, Iron, RyhB, Fur, luxS, Al-2, Virulence factors

Posted Date: September 29th, 2021

DOI: https://doi.org/10.21203/rs.3.rs-936419/v1

License: (9) This work is licensed under a Creative Commons Attribution 4.0 International License.

Read Full License

Version of Record: A version of this preprint was published at Scientific Reports on January 17th, 2022. See the published version at https://doi.org/10.1038/s41598-021-04757-9. 


\title{
Quorum Sensing and Iron-Dependent Coordinated Control of Autoinducer-2 Production via Small RNA RyhB in Vibrio vulnificus
}

\author{
Keun-Woo Lee, Yancheng Wen*, Na-Young Park, Kun-Soo Kim
}

Department of Life Sciences, Sogang University, Seoul, Korea.

Address correspondence to Kun-Soo Kim, Department of Life Science, Sogang University, Baekbeom-Ro, Mapo-Gu, Seoul 121-742, Korea; Tel.: (822)-705-8460; E-mail:

\section{kskim@,sogang.ac.kr}

"Present address: Key Laboratory of Ministry of Education for Gastrointestinal Cancer Research Center for Molecular Medicine, Fujian Medical University, Fuzhou, Fujian, People’s Republic of China.

Keywords: Vibrio vulnificus, Quorum-sensing, Iron, RyhB, Fur, luxS, AI-2, Virulence factors 
Roles for the non-coding small RNA RyhB in quorum-sensing and iron-dependent gene modulation in the human pathogen $V$. vulnificus were assessed in this study. Both the quorum sensing master regulator SmcR and the Fur-iron complex were observed to bind to the region upstream of the non-coding small RNA RyhB gene to repress expression, which suggests that RyhB is associated with both quorum-sensing and iron-dependent signaling in this pathogen. We found that expression of LuxS, which is responsible for the biosynthesis of autoinducer-2 (AI-2), was higher in wild type than in a ryhB-deletion isotype. RyhB binds directly to the $5^{\prime}-$ UTR of the luxS transcript to form a heteroduplex, which not only stabilizes LuxS mRNA but also disrupts the secondary structure that normally obscures the translational start codon and thereby allows translation of LuxS to begin. The binding of RyhB to LuxS mRNA requires the chaperone protein Hfq, which stabilizes RyhB. These results demonstrate that the small RNA RyhB is a key element associated with feedback control of AI-2 production, and that it inhibits quorum-sensing signaling in an iron-dependent manner. This study, taken together with previous studies, shows that iron availability and cell density signals are funneled to SmcR and RyhB, and that these regulators coordinate cognate signal pathways that result in the proper balance of protein expression in response to environmental conditions. INTRODUCTION

Bacterial pathogens are exposed to a variety of stressors either in the natural environment or in the host environment during the infection process, such as nutrient restrictions, temperature changes, osmotic stress, and oxidative stress ${ }^{1}$. These organisms have evolved sophisticated 
mechanisms to control gene expression in these diverse environments by sensing relevant environmental factors and rapidly adapting to improve both survival and pathogenicity. It is well known that iron plays an important role in regulating virulence factors in pathogenic bacteria $^{2}$. Fur, ferric uptake regulator, is a well-studied regulatory factor that controls the expression of numerous genes in association with iron ${ }^{3,4}$. Similar examples are documented for V. vulnificus: Fur increases the expression of heme receptors in the presence of heme ${ }^{5}$, Fur represses genes encoding the biosynthesis and uptake of the siderophore vulnibactin ${ }^{6}$, and Fur suppresses quorum-sensing by modulating both the master regulator $s m c R$ and Qrrs, the quorum-sensing small regulatory $\mathrm{RNAs}^{7,8}$.

Bacterial cell density is another important factor influencing a wide range of cellular activities including the virulence of pathogenic bacteria. Regulation in response to cell density is achieved through a quorum-sensing pathway that monitors the accumulation of small diffusible signaling molecules produced by cognate cells in a closed space at high cell density, and this subsequently influences the expression of genes related to numerous functions such as survival, biofilm formation, and virulence ${ }^{9-11}$. The quorum-sensing pathway in $V$. vulnificus is similar to that of Vibrio harveyi and Vibrio cholerae. V. vulnificus harbors a homolog of $V$. harveyi luxS, which encodes an enzyme for the biosynthesis of the autoinducer-2 signaling molecule ${ }^{12,13}$. The genome sequence of the well-studied $V$. vulnificus strain MO6-24/O (GenBank $^{\mathrm{TM}}$ accession number CP002469.1 for chromosome I and CP002470.1 for chromosome II $)^{14}$ revealed that there is no biosynthetic gene for autoinducer-1 (AI-1; homoserine lactone molecule) or cholera autoinducer-1 (CAI-1). However, LuxPQ, the cognate receptor for autoinducer-2 (AI-2) and homologues of LuxU and LuxO involved in a phosphorelay in $V$. harveyi ${ }^{15-19}$ have been identified. The AI-2 signal converges to LuxO, a nitrogen 
regulatory protein $(\mathrm{NtrC})$ homolog, and is transduced to the master regulator $\mathrm{SmcR}^{20-22}$. In addition, this pathogen harbors yet another quorum-sensing pathway that is mediated by cyclic dipeptide ${ }^{23-25}$.

RyhB, a non-translated small RNA, was first identified as important for iron metabolism in E. coli $^{26}$. Since that time, genes homologous to RyhB have been found and characterized in the context of the iron regulon in many other bacteria such as V.cholerae, Salmonella typhimurium, Yersinia pestis, and Shigella dysenteriae ${ }^{27-30}$. Transcription is negatively regulated by Fur in the presence of iron. Under iron-limiting conditions, this repression is relieved and RyhB then represses genes encoding iron-containing proteins, such as superoxide dismutase and succinate dehydrogenase $^{26,31}$. In addition, RyhB also activates the expression of $s h i A$, a gene encoding shikimate permease, which is associated with siderophore synthesis, by directly pairing with the 5'-untranslated region of the shiA mRNA in E. coli ${ }^{32}$. RyhB also translationally downregulates fur expression ${ }^{33}$. In $V$. cholerae, a ryhB mutant showed decreased mobility and poor biofilm formation compared to wild type ${ }^{27}$. RyhB represses SodB expression by pairing with the 5'-untranslated region of the SodB mRNA and causing the coupled degradation induced by $\mathrm{RNase}^{34,35}$. The sRNA chaperone Hfq is essential for this process as it binds and protects RyhB from RNase E degradation, thereby extending its half-life $\mathrm{e}^{34,36-38}$.

Iron is essential for the growth of living organisms, but low solubility in the environment of a living cell makes this element a limiting factor for growth. High cell density leads to an even more severe iron stringency. Meanwhile, when intracellular iron is in excess, radicals that compromise the viability of cells are generated ${ }^{39}$. Therefore, it is expected that cell density and iron availability are intertwined and that these factors work together to control the expression of related genes. Here we report a new connection between quorum-sensing and the iron stress 
response mediated by the small non-coding RNA RyhB in $V$. vulnificus. The results of this study provide evidence for a close relationship between iron levels and cell density in terms of gene regulation and also add to the increasingly long list of roles for non-translated small RNA in pathogenic bacteria.

\section{RESULTS}

\section{Identification of the transcription start site of RyhB and evidence for Hfq-dependent}

stabilization of the RNA. Our previous study showing the repression of $s m c R$ by the Fur-iron complex in $\mathrm{V}_{\text {vulnificus }}{ }^{7}$ led us to further investigate the relationship between quorum-sensing and iron in this pathogen. In both E. coli and V. cholerae, the Fur-iron complex represses ryhB, a gene encoding a small $\mathrm{RNA}^{26-28}$. Therefore we speculated that if any small RNAs affect quorum-sensing in an iron-dependent manner in $V$. vulnificus, the most likely candidate would be RyhB. A search of the genome sequence of $V$. vulnificus revealed a 224-bp gene with 56\% similarity to the ryhB of $V$. cholerae. This gene mapped between the genes encoding DNA polymerase I (GenBank ${ }^{\mathrm{TM}}$ accession number VVMO6_02895) and porphobilinogen (VVMO6_02896). The transcription start site was identified through primer extension experiments and typical -10 and -35 consensus sequences also were observed to be present (Fig. $\mathrm{S} 1)$. The chaperone Hfq is required to stabilize RyhB in E. coli ${ }^{35}$. Therefore, we looked for a similar role in $V$. vulnificus and compared levels of the RyhB transcript in wild type and a $\Delta h f q$ mutant at several time points after rifampicin was added to pause transcription. As shown in Figure 1, RyhB from wild type is still detected up to 30 minutes after rifampicin treatment with a half-life of about 30.7 minutes. On the other hand, in the $h f q$ mutant RyhB was degraded 
quickly with a half-life of about 7.5 minutes, and was barely detectable at 15 minutes following the rifampicin treatment (Fig. 1).

Fur represses RyhB expression in the presence of iron. We assessed the expression levels of $r y h B$ under different iron conditions. RNA was extracted from $V$. vulnificus grown in either iron rich medium ( $\mathrm{LB}$ broth with or without $25 \mu \mathrm{M}$ of $\mathrm{FeSO}_{4}$ ) or under iron limiting conditions (LB broth with $200 \mu \mathrm{M}$ of 2, 2'-dipyridyl) (Fig. 2a). In wild type MO6-24/O cells, the ryhB transcript is barely detectable in cells grown in LB broth with or without $25 \mu \mathrm{M}$ of $\mathrm{FeSO}_{4}$, while an abundance of transcript was detected when the iron chelator 2, 2'-dipyridyl was added. In contrast, a fur-deletion mutant $(\Delta f u r)$ had high amounts of the RyhB transcript regardless of iron concentrations. These results suggest that iron strongly represses the transcription of $r y h B$ and that Fur is necessary for this repression.

Fur regulates gene expression in response to environmental iron conditions by binding to a specific site called the 'Fur box' in the promoter region of target genes ${ }^{6,40,41}$. To verify that Fur regulates $r y h B$ expression in a similar manner, a gel-mobility shift assay was performed using a DNA fragment containing the region upstream to $r y h B$ and purified Fur protein in the presence of the divalent ion $\mathrm{Mn}^{2+}$ (Fig. 2b). Fur specifically bound to this DNA sequence in the presence $\mathrm{MnSO}_{4}$ and binding was abolished if EDTA was added to sequester the divalent ion (Fig. 2c). The binding site for Fur within the $r y h B$ promoter region was defined through a DNase I footprinting assay in the presence of $100 \mu \mathrm{M} \mathrm{MnSO}_{4}$ (Fig. S2). The result showed that Fur protected a region from -64 to +6 with respect to the transcription start site, covering the consensus -10 and -35 promoter sequences (Fig. S3). 
133 SmcR represses transcription of $r y h B$ by binding to the promoter region. Our previous

134 study showed that regulation of $v v s A B$, which encodes a biosynthetic enzyme for the siderophore vulnibactin, is coordinately regulated by both iron and quorum-sensing ${ }^{6}$. Furthermore, expression of the qrr genes, encoding small RNAs associated with quorumsensing modulation ${ }^{9}$, also are regulated by iron $^{8}$. These results point to a connection between RyhB and quorum-sensing regulation and that these are somehow associated with iron levels. In support of this prediction, analysis of the nucleotide sequences upstream of $r y h B$ revealed similarities to the consensus binding motif for the quorum-sensing master regulator $\mathrm{Smc} \mathrm{R}^{42}$ (Fig. S3).

To verify this, we measured the expression of $r y h B$ using a $\operatorname{lux} A B$ transcriptional fusion as a reporter in the following strains: wild type MO6-24/O; a mutant with a deletion in luxO $(\Delta \operatorname{luxO})$ such that it lacks the cytoplasmic signal transducer that degrades SmcR at low cell density via Qrr and $\mathrm{Hfq}^{8}$; an $s m c R$-deletion mutant $(\Delta s m c R)$; and a luxOsmcR double mutant $(\Delta l u x O \Delta s m c R)$. In both $\Delta s m c R$ and $\Delta l u x O \Delta s m c R$, transcription of $r y h B$ was significantly higher than that of wild type at early stationary phase $\left(\mathrm{A}_{600} \cong 1.0\right)$ (Fig. 3). Conversely, in a $\Delta l u x O$ mutant, transcription was just half that of wild type. These results indicated that SmcR represses $r y h B$ expression at the transcriptional level. A DNA fragment containing the upstream region of $r y h B$ was incubated with increasing amounts of purified SmcR and analyzed in a gel shift assay (Fig. 4a). As expected, SmcR bound directly to the promoter region of $r y h B$. DNase I footprinting identified the binding region as -27 to +1 with respect to the transcriptional start site, including a putative -10 promoter sequence (Fig. $4 \mathrm{~b}$ and Fig. S3). These results indicate 
to prevent the binding of RNA polymerase.

RyhB promotes the production of AI-2 in $\boldsymbol{V}$. vulnificus. The results described above suggested that RyhB is involved in the iron-dependent regulation of the quorum-sensing pathway in $V$. vulnificus. To examine this further, we compared the transcription of genes related to the quorum-sensing pathway in wild-type and a ryhB-deletion mutant using transcriptome analysis. Expression levels of luxPQUO and SmcR were not significantly different in the two isotypes. However, the expression of luxS, encoding an AI-2 biosynthetic enzyme, was approximately $40 \%$ lower in a $\Delta r y h B$ mutant than in wild type (Table S3). To

verify this result, we compared both AI-2 production and LuxS expression in wild type and $\Delta r y h B$ mutant backgrounds. Supernatants from each of the two $V$. vulnificus strains cultured in $\mathrm{AB}$ minimal medium with a low iron concentration were assessed for the production of $\mathrm{AI}-2$ using the bio-indicator $V$. harveyi $\mathrm{BB} 170^{43}$ and measuring luminescence. The luminescence induced by the wild type culture supernatant was significantly higher than that of $\Delta r y h B$, and introduction of an exogenous $r y h B$-expressing clone into $\Delta r y h B$ in trans restored luminescence to wild type levels, whereas introduction of vector alone did not (Fig. 5a). These results indicate that RyhB promotes expression of LuxS under iron-limiting conditions. We then assessed the effects of RyhB on the expression of a gene normally regulated by the AI-2 quorum-sensing system. We quantitatively measured the expression of $v v p E$, which encodes elastase, a virulence factor that is positively regulated by quorum sensing ${ }^{7,}{ }^{44}$. Iron repressed $v v p E$ expression regardless of RyhB. However, in the absence of iron, expression in the $\Delta r y h B$ mutant was significantly lower than in wild type (Fig. 5b). This result supports the idea that enhanced production of AI-2 by RyhB promotes quorum-sensing signaling. 
In summary, these results indicate that RyhB increases AI-2 mediated quorum-sensing signaling by enhancing the production of AI-2 itself, and that SmcR represses RyhB as a mechanism for feedback inhibition to repress AI-2 production. The presence of iron, as part of the Fur-iron complex, down-regulates overall quorum-sensing signaling by inhibiting the transcription of both SmcR and RyhB.

RyhB delays the decay of LuxS mRNA by binding directly to the $5^{\prime}$-UTR of LuxS. The next question was how RyhB enhances the production of AI-2 at the molecular level. The noncoding sRNA RyhB post-transcriptionally regulates gene expression by affecting the turnover of mRNAs in $E$. $\operatorname{coli}^{26}$. We assumed that RyhB would affect the luxS mRNA in the same manner. To test this, levels of the LuxS transcript were measured over time after cells were treated with rifampicin. As shown in Figures 6a and 6b, LuxS mRNA degraded faster in $\Delta r y h B$ than in wild type. Previous studies reported that Hfq is required for the functioning of RyhB. To test for this possibility in $V$. vulnificus, we compared the levels of LuxS mRNA following rifampicin treatment in wild type, $\Delta r y h B, \Delta h f q$, and a $\Delta r y h B \Delta h f q$ double mutant. As shown in Figure $6 \mathrm{c}$, in the absence of Hfq, luxS mRNA levels decreased significantly independent of RyhB, indicating that even though RyhB appears to stabilize LuxS mRNA, this function requires Hfq. Generally, non-coding RNAs modulate gene expression by base pairing with target mRNAs at the $5^{\prime}$-UTR (untranslated region) ${ }^{45}$, and the sRNA chaperone Hfq either stabilizes the sRNA or facilitates binding of the sRNA to the target ${ }^{46}$. We predicted that RyhB delays the decay of LuxS mRNA by binding directly to the $5^{\prime}$-UTR of LuxS. A ${ }^{32}$ P-labeled 102-bp LuxS RNA fragment including bases -62 to +40 with respect to the $\operatorname{lux} S$ translation start site was expressed 
by in vitro transcription using T7 RNA polymerase. Full-length RyhB was also transcribed using in vitro transcription, and increasing amounts of this transcript were incubated with the labeled LuxS mRNA probe in the presence or absence of Hfq. In the presence of Hfq, RyhB bound to LuxS 5'-UTR in a concentration-dependent manner (Fig. 7a), whereas no binding was observed in the absence of Hfq. We also assessed the effect of Hfq on the half-life of $r y h B$ mRNA and observed that stability was significantly reduced in $\Delta h f q$ compared to wild type (Fig. 7b). Together these indicate that RyhB binds directly to the $5^{\prime}$-UTR of LuxS mRNA and stabilizes it with the assistance of Hfq.

Confirmation of base pairing between RyhB and the 5'-UTR of LuxS. The mFold software ${ }^{47}$ was used to predict secondary structures that may form in the 5'-UTR of LuxS (Fig. 8a), one of which is a stem-and-loop structure (SL2) that would obscure the start codon. Hybridization between RyhB and the 5'-UTR of LuxS was predicted to include base pairing in three regions labeled HR (Hybridized Region) 1, 2, and 3 (Fig. 8b). If hybridization occurs at these sites, Loops SL1 and SL2 would be resolved and the start codon of LuxS mRNA would be exposed (Fig. 8b).

To confirm these predictions, we performed a primer extension in the presence or absence of LuxS 5'-UTR by reverse transcription using RyhB RNA as a template and primer RyhB-PE (Table S3, Fig. S3) which is complementary to the 3'-end of RyhB RNA. We expected that binding of RyhB to the LuxS mRNA in the presence of Hfq would produce shorter immature cDNA products due to the formation of secondary structures between two RNA molecules that partially block the primer extension. In fact, we observed partially extended cDNA molecules 
that terminated at cytosine at position 108 and uracil at position 84 relative to the first base of the $r y h B$ transcript (Fig. 9a and 9b). These two sites correspond to the $3^{\prime}$-end of the HR2 and HR3 regions of RyhB, respectively. cDNA terminated at adenine at position 97 also was observed. This residue is the first base at the $3^{\prime}$-end of Loop 2 (Fig. 9b). These results suggested that hybridization to LuxS mRNA does indeed occur at HR2 and HR3. We speculate that there may be weak hybridization at HR1 such that termination of cDNA at this region was barely detectable. To confirm the involvement of the HR2 and 3 regions of $r y h B$ in regulation of the LuxS expression, we constructed derivatives of RyhB with mutations in either or both of HR2 or 3 (named HR2m, HR3m, and HR2\&3m) by site-directed mutagenesis. A mutation called HR0m at a site outside of the hybridized region (HR0) was generated as a positive control (Fig. $9 \mathrm{~b}$ and Fig. S3).

Wild type RyhB and each of the RyhB mutations (HR0m, 2m, 3m, and $2 \& 3 \mathrm{~m}$ ) were introduced into a $r y h B$-null mutant strain $(\Delta r y h B)$ and assessed for both AI-2 production using the $V$. harveyi indicator strain BB170 (Fig. 10a) and levels of LuxS by western hybridization using antibody against purified LuxS (Fig. 10b). RyhB with HR0m did not differ significantly from wild type. However, the presence of either HR2m or $3 \mathrm{~m}$ led to significant decreases in both AI-2 production and LuxS expression, suggesting that HR2 and 3 are critical for LuxS regulation. However, the results of $\operatorname{lux} S$ qRT-PCR for wild type and the $r y h B$ mutants showed that these mutations did not affect transcription levels of $\operatorname{lux} S$ under experimental conditions (Fig. 10c), suggesting that the regulation of the LuxS expression by RyhB is exerted at the translational level. 
245 It is well known that an adequate concentration of iron is required for the survival of bacteria ${ }^{2}$, ${ }^{48}$. Nevertheless, iron is not readily available in natural environments due to its low solubility at neutral $\mathrm{pH}^{49}$. Furthermore, it is very difficult for pathogenic bacteria to compete with host cells for iron ${ }^{50}$. Therefore, pathogenic bacteria are equipped with various mechanisms to more effectively scavenge iron in the host environment. Our previous studies showed that iron levels affect quorum-sensing pathways ${ }^{6,8}$. This study more specifically defines the relationship between iron levels and quorum-sensing by showing that iron inhibits the production of AI-2, an initiation signal for quorum-sensing. Our observations lead us to propose that one of the most important roles for quorum-sensing is to control the timing of expression of virulence factors in pathogens as they prepare to attack host cells to acquire nutrients such as iron.

Fur is the most common regulator of iron-dependent target genes. However, neither genetic analysis using a reporter fusion to $\operatorname{luxS}$ nor gel-shift assays using purified Fur-iron along with the upstream region of $\operatorname{luxS}$ provided any evidence that Fur directly controls $l u x S$ expression in $V$. vulnificus (data not shown). Interestingly, unlike other components of the quorum-sensing pathway, luxS expression appears to be controlled by RyhB at the translational level. This mode of regulation may allow for a continuous basal level of AI-2 expression even in the presence of iron rather than the tight suppression that may occur if Fur were involved. If cells do not produce any AI-2 at all, the quorum-sensing pathway cannot quickly resume once it is shut down by the Fur-iron complex. This scenario may also explain the presence of the strong promoter sequence of $r y h B$, which is very similar to the canonical consensus promoter sequence in this organism. In this way, expression of $r y h B$ is initiated swiftly once the repression exerted by Fur-iron is relieved. Employing RyhB may also provide a particular 
advantage in that this small RNA functions at the translational level to alter expression quickly and, due to the short half-life of the molecule, repression is rapidly relieved if the SmcR or Furiron signals disappear. The chaperone $\mathrm{Hfq}$ is absolutely required for RyhB activity. Furthermore, levels of LuxS mRNA in a $h f q$-deletion mutant were even lower than in a $r y h B$ deletion isotype (Fig. 6c), suggesting that yet another factor(s) may exist, possibly an additional small RNA specific to $\operatorname{luxS}$. A search for non-coding small RNAs modulated by RyhB may provide more insight into the iron-dependent modulation of genes.

Quorum-sensing signaling pathways are a valuable way for pathogenic bacteria to adapt to host environmental conditions but they are energetically expensive due to the numerous factors and variety of regulatory mechanisms that are employed. Therefore, it is necessary for cells to be equipped with the ability to control this signaling when it is not needed. For example, once a quorum-sensing signaling molecule accumulates within a closed environment, it is possible that cell would continue to sense the signal even when expression of target genes are no longer required. The best way to avoid such a waste of energy may be lower the concentration of signaling molecules. Two possible mechanisms are either to degrade the signal molecule or to cease synthesis of the signal molecule. It is known that bacteria harbor enzymes to degrade the homoserine lactone signal molecule ${ }^{51,52}$. However, whether the AI-2 molecule is degraded in a similar way remains to be elucidated. To the best of our knowledge, ours is the first study to show that feedback inhibition of quorum signaling occurs through the inhibition of AI-2 synthesis. Other small RNAs called Qrrs are also known to be involved in the feedback control of quorum-sensing ${ }^{53,54}$. However, these appear to be involved in elaborate control of the signal to properly adapt to changes in cell density and not in feedback control of the quorum-sensing pathway as a whole. 
AI-2 is a signal that plays a very important role in bacterial community structure, affecting inter-species communication as well as intra-species communication ${ }^{9}$. AI-2 signaling from pathogenic bacteria can be transmitted to other recognizable bacteria, and a decrease in AI-2 levels in response to the presence of iron can certainly affect other microbiota present in the infected regions of a host. A comparison of the gut microbiota in mice infected with wild type V. vulnificus and mice infected with a luxS-deleted isotype are significantly discernable (our unpublished data). This suggests that the presence of iron alone can indeed affect the community structure of the microbiota, in addition to influencing levels of the quorum-sensing signal molecules and related target functions. Studies on the effect of AI-2 on host microbiota in the context of iron concentrations may provide interesting insight into host-microbe interactions.

The nucleotide sequences of RyhB and LuxS mRNAs and possible hybridization between these two molecules in three related Vibrio spp. ( $V$. cholerae, $V$. parahaemolyticus, and $V$. harveyi) demonstrated that, for all three, the start codon for $\operatorname{lux} S$ is hidden within stem-loop structures unless binding with RyhB resolves this secondary structure (Fig. S4), implying that this type of regulation is common among Vibrionaceae. Considering that major virulence process.

Iron, or the molecules with which it interacts, may be good targets for the development of agents to control pathogenic bacteria. This study demonstrated that iron inhibits the quorum sensing signaling pathway associated with expression of virulence factors, and any tactics to enhance iron solubility may be of value to control pathogenic bacteria. Development of 
molecules such as anti-sense RNAs that interfere with the action of RyhB or possibly direct inhibitors of Hfq may be useful clinical approaches.

\section{MATERIALS AND METHODS}

Strains and culture condition. Strains and plasmids used in this study are listed in Table S1. Escherichia coli cells were cultured in Luria-Bertani (LB) medium at $37^{\circ} \mathrm{C}$. V. vulnificus strains were grown at $30^{\circ} \mathrm{C}$ in $\mathrm{LB}$ medium supplemented with $2.0 \%$ (w/v) $\mathrm{NaCl}$ (LBS) or in $\mathrm{AB}$ minimal medium $\left(0.3 \mathrm{M} \mathrm{NaCl}, 0.05 \mathrm{M} \mathrm{MgSO}_{4}, 0.2 \%\right.$ casamino acid, $1 \mathrm{mM} \mathrm{KPO} 4,1 \mathrm{mM} \mathrm{L}$ arginine, $\mathrm{pH} 7.5)^{55}$. All media components were purchased from Difco (Detroit, USA), and antibiotics were purchased from Sigma (St. Louis, USA).

Determination of the transcription start site of $\boldsymbol{r y h} \boldsymbol{B}$. RNA was isolated from $V$. vulnificus using the RNase Easy Mini Kit (Qiagen, Valencia, CA), and RNA concentration was determined using a Biophotometer (Eppendorf, Hamburg, Germany). A 500 ng sample of RNA extracted from $V$. vulnificus was incubated with $5^{\prime}$-labeled primer RyhB-PE at $65^{\circ} \mathrm{C}$ and chilled on ice. Reverse transcription was performed using a PrimeScript RT reagent kit (Takara, Tokyo, Japan). The resulting product and sequencing ladder were resolved on a $6 \%$ polyacrylamide sequencing gel to identify the transcription start site.

Construction of $\boldsymbol{r y h B}$ deletions in $\boldsymbol{V}$. vulnificus. A DNA fragment comprising the upstream region of $r y h B$ was amplified by primers RyhB-KO-upF and RyhB-KO-upR, and ligated into 
a pGEM-T easy vector, generating plasmid pGEM-T-RyhBup. The downstream region of $r y h B$ was amplified by primers RyhB-KO-downF and RyhB-KO-downR and ligated into a pGEMT easy vector, generating plasmid pGEM-T-RyhBdown. Plasmid pGEM-T-RyhBup was digested with restriction enzymes PstI and BamHI, and the resulting DNA fragment containing the $r y h B$ upstream region was ligated into plasmid pGEM-T-RyhBdown to construct pGEMT-RyhBKO. The construction was digested with $X h o \mathrm{I}$ and $X b a \mathrm{I}$, and subsequently ligated into pDM4 to obtain pDM4-RyhBKO which was subsequently introduced into E. coli S17-1 $\lambda$ pir to be mobilized into $V$. vulnificus by conjugation. Double crossover selection was performed on a $10 \%$ sucrose plate as described previously ${ }^{56}$. The $r y h B$ deletion mutant, $\Delta r y h B$, was confirmed by PCR and DNA sequencing.

Gel shift assay. A 336-bp DNA fragment including the $r y h B$ promoter region $(-213$ to +123 with respect to the transcriptional start site) was PCR-amplified using the ${ }^{32} \mathrm{P}$-labeled primers RyhB-F1 and RyhB-R1 (Table S2). For the gel shift assay, 10 ng of labeled DNA fragment was incubated with increasing amounts of purified SmcR $(0$ to $1 \mu \mathrm{M})$ or Fur $(0$ to $1 \mu \mathrm{M})$ in a $20 \mu \mathrm{l}$ reaction for 30 minutes at $37^{\circ} \mathrm{C}$. The SmcR binding reaction buffer contained $10 \mathrm{mM}$ HEPES, $100 \mathrm{mM} \mathrm{KCl}, 200 \mu \mathrm{M}$ EDTA, and 10\% glycerol at $\mathrm{pH}$ 7.5. The Fur binding reaction contained $10 \mathrm{mM}$ HEPES, $100 \mathrm{mM} \mathrm{KCl}$, and 10\% glycerol at $\mathrm{pH} 7.5$ and was supplemented with either $100 \mu \mathrm{M} \mathrm{MnSO}_{4}$ or $1 \mathrm{mM}$ EDTA. The binding reaction was terminated by the addition of $3 \mu 1$ sucrose dye solution $(0.25 \%$ bromophenol blue, $0.25 \%$ xylene cyanol, $40 \%$ sucrose $)$ and the samples were resolved on a 6\% neutral polyacrylamide gel. For gel shift assays of RyhB and LuxS 5'-UTR, 10 ng of ${ }^{32} \mathrm{P}$-labeled LuxS 5'-UTR and increasing amounts of RyhB were incubated at $70^{\circ} \mathrm{C}$ for 10 minutes and then chilled on ice for at least 1 minute. Then $2 \mu 1$ of $10 \times$ 
structure buffer (100 mM Tris-Cl, $50 \mathrm{mM}$ magnesium acetate, $1 \mathrm{M}$ ammonium chloride, $5 \mathrm{mM}$ DTT, $\mathrm{pH}$ 7.5) and Hfq were added and incubated at $30^{\circ} \mathrm{C}$ for 10 minutes. The binding reaction was terminated by the addition of $4 \mu \mathrm{l}$ of sucrose dye and resolved on a $6 \%$ neutral polyacrylamide gel.

DNase I footprinting assay for binding of SmcR and Fur to the upstream region of $r y h B$. 
378 An end-labeled 340-bp DNA fragment consisting of bases -217 to +123 relative to the transcription start site of $r y h B$ was generated by PCR amplification using primers ${ }^{32} \mathrm{P}-$ labeled RyhB-F1 and RyhB-R1 (Table S2). To determine the binding site of SmcR, 200 ng of the amplified DNA fragments was incubated with increasing amounts of purified SmcR or Fur for 30 minutes at $37^{\circ} \mathrm{C}$ in $50 \mu \mathrm{l}$ buffer $(10 \mathrm{mM}$ HEPES, $100 \mathrm{mM} \mathrm{KCl}, 200 \mu \mathrm{M}$ EDTA, $10 \%$ glycerol, $\mathrm{pH}$ 7.5). To identify the Fur binding region, $200 \mathrm{ng}$ of the resulting DNA fragments was incubated for 30 minutes with increasing amount of purified Fur in $50 \mu 1$ of binding solution (10 mM HEPES, 100 mM KCl, 10\% glycerol, $100 \mu \mathrm{M} \mathrm{MnSO}_{4}$, $\mathrm{pH}$ 7.5). After supplementing $50 \mu \mathrm{l}$ of $\mathrm{CaCl}_{2}-\mathrm{MgCl}_{2}$ solution $\left(5 \mathrm{mM} \mathrm{CaCl}_{2}, 10 \mathrm{mM} \mathrm{MgCl}_{2}\right)$ to the binding reaction, 0.25 unit of DNase I (Promega, Madison, USA) was added and allowed to react for 1 minute at $37^{\circ} \mathrm{C}$ before terminating with $90 \mu \mathrm{l}$ stop solution (200 mM NaCl, $30 \mathrm{mM}$ EDTA, 1\% SDS). To precipitate the DNA, $500 \mu$ l ethanol was added and samples were incubated on ice for 30 minutes, after which pellets were collected, washed with $70 \%$ ethanol, and suspended in $10 \mu 1$ loading buffer ( $98 \%$ formamide, $0.1 \%$ xylene cyanol, $0.1 \%$ bromophenol blue). The resulting product and sequencing ladder generated with the ${ }^{32} \mathrm{P}-$ labled RyhB-F1 primer (Table S2) were resolved on a $6 \%$ polyacrylamide sequencing gel.

Detection of AI-2 production. The AI-2 assay was performed using the $V$. harveyi reporter strain BB170 as previously described ${ }^{43}$. Briefly, BB170 was prepared by culturing overnight in $\mathrm{LB}$ broth at $30^{\circ} \mathrm{C}$, then washing twice and diluting 1:3000 in fresh $\mathrm{AB}$ minimal medium. Test cultures of $V$. vulnificus were grown overnight in LBS broth and then washed once before diluting 1:250 in fresh $\mathrm{AB}$ broth. Each hour, $\mathrm{A}_{600}$ was measured and $10 \mu \mathrm{l}$ of cell-free supernatant was collected and mixed with $90 \mu$ l of diluted BB170, prepared as described above, 
401

402

403

404

405

406

407

408

409

410

411

412

413

414

415

416

417

418

419

420

421

in 96 well plates at $30^{\circ} \mathrm{C}$. The luminescence was measured using a Mithras LB 940 multimode microplate reader (Berthold, Germany).

RNA synthesis by in vitro transcription. Template DNA of ryhB and $\operatorname{luxS}$ was prepared for in vitro transcription by PCR using primers containing the $\mathrm{T} 7$ promoter sequences as shown in Table S2. RNA was synthesized by in vitro transcription using T7 RNA polymerase (Takara, Tokyo, Japan) using the prepared template DNA at $37^{\circ} \mathrm{C}$ following the protocol provided and was further purified using a Monarch ${ }^{\circledR}$ RNA Cleanup Kit (NEB, Cambridge, USA).

\section{Determination of the regions of RyhB RNA and LuxS mRNA that hybridize with each} other using primer extension with reverse transcriptase. RyhB (50 ng) was incubated with LuxS 5'-UTR and ${ }^{32} \mathrm{P}$-labeled primer RyhB-PE, which is complementary to RyhB from residues 167 and 189 relative to the transcription start site of $r y h B$, at $65^{\circ} \mathrm{C}$ for 10 minutes and then chilled on ice. Hfq and $10 \times$ structure buffer $(100 \mathrm{mM}$ Tris-Cl, $50 \mathrm{mM}$ magnesium acetate, $1 \mathrm{M}$ ammonium chloride, $5 \mathrm{mM}$ DTT, $\mathrm{pH}$ 7.5) were added and the mixture was incubated at $30^{\circ} \mathrm{C}$ for 10 minutes. Lastly, dNTPs and 1 unit of SuperScript ${ }^{\mathrm{TM}}$ III reverse transcriptase (Invitrogen, Carsbad, USA) were added and incubated at $30^{\circ} \mathrm{C}$ for 1 hour. Reactions were terminated at $85^{\circ} \mathrm{C}$ for 5 minutes, and samples were resolved on a $6 \%$ polyacrylamide sequencing gel alongside a sequencing ladder generated by the same primer.

Purification of LuxS and Hfq proteins. A DNA fragment covering the 519-bp luxS open 
reading frame (ORF) encoding the 172-amino acids of LuxS was PCR-amplified using primers STREP-LUXSF and STREP-LUXSR (Table S2). The amplified fragment was sub-cloned into pASK-IBA-7 (IBA, Göttingen, Germany) which generates a fusion between the Strep-tag and the N-terminus of the expression protein. The resulting Strep-tagged luxS construct was transformed into E. coli BL21 (DE3) (Novagen, Madison, USA), and over-expressed by inducing with $10 \mu \mathrm{g} / \mathrm{ml}$ anhydrotetracycline. The bacterial pellets were suspended in $\mathrm{W}$ buffer (100 mM Tris-Cl, $150 \mathrm{mM} \mathrm{NaCl}, \mathrm{pH} 8.0$ ), sonicated, and then centrifuged at 13,000 rpm for 15 minutes. The resulting supernatant was applied to $1 \mathrm{ml}$ of Strep-Tactin-Sepharose resin (IBA, Göttingen, Germany) and bound proteins were eluted with E buffer (100 mM Tris-Cl, $150 \mathrm{mM}$ $\mathrm{NaCl}, 1 \mathrm{mM}$ EDTA, $2.5 \mathrm{mM}$ desthiobiotin, $\mathrm{pH}$ 8.0). The eluted proteins were assessed for purity using 15\% sodium dodecyl sulfate-polyacrylamide gel electrophoresis (SDS-PAGE). To purify the Hfq protein, DNA spanning the 261-bp Hfq ORF encoding 86 amino acids of Hfq was PCR-amplified using primers STREP-HFQF and STREP-HFQR (Table S2). The amplified fragment was cloned into pASK-IBA-7 to generate pASK-IBA-Hfq (Table S1), and Hfq was purified as described above.

Construction of a $r y h B$-deletion and series of site-directed mutations in $r y h B$. A DNA fragment containing the complete sequence of $r y h B$ was PCR-amplified using primers CryhBF and CryhB-R (Table S2). The resulting product was ligated into pGEM-T easy vector (Promega). After confirmation by sequencing, the DNA fragment containing the $r y h B$ sequence was cut with $K p n I$ and $X b a I$ and cloned into pRK415, generating pRK-RyhB. Nucleotides that potentially bind to LuxS 5'-UTR were mutated using the EZchange ${ }^{\mathrm{TM}}$ Site-directed Mutagenesis Kit (Enzynomics, Deajeon, Korea). Primers ryhB_SDM2_F and ryhB_SDM2_R 페이지 20 / 36 
were used to construct pRK-RyhB2m; primers ryhB_SDM3_F and ryhB_SDM3_R were used to construct pRK-RyhB3m; all four primers (ryhB_SDM2_F\&R, ryhB_SDM3_F\&R) were 447 used to construct pRK-RyhB2\&3m; and primers ryhB_SDM_CON_F and ryhB_SDM_CON_R were used to construct the control pRK-RyhB0m (Table S2). The resulting constructs were mobilized from S17-1 to mutant strain $\Delta r y h B$. Conjugants were selected in thiosulfate-citrate-bile salts-sucrose agar (TCBS) plate medium supplemented with $1 \mu \mathrm{g} / \mathrm{ml}$ tetracycline.

\section{Preparation of polyclonal rabbit antibody against purified LuxS and western}

hybridization. Purified LuxS was used to produce polyclonal rabbit antibodies (Ab Frontier,

Seoul, South Korea). For LuxS expression western blot analysis, $V$. vulnificus MO6-24/O wild type or mutants were cultured in LBS broth for 6 hours, then diluted 1:100 in fresh LB broth. After 3 hours of growth, cells were collected and washed twice with PBS, after which $20 \mu \mathrm{g}$ of each lysate was resolved by SDS-PAGE and transferred to Hybond P membrane (Amersham, Arlington Heights, USA). The membrane was incubated first with polyclonal rabbit antibodies against LuxS (1:2000) and then with goat anti-rabbit IgG-AP (1:5000) (Santa Cruz Biotechnology, CA, USA). LuxS expression was visualized using Western blotting luminal reagent (Santa Cruz Biotechnology, CA, USA).

\section{REFERENCES}


466 Press $2^{\text {nd }}$ edition (2010).

467 2. Ratledge, C. \& Dover, L. G. Iron metabolism in pathogenic bacteria. Annu. Rev. Microbiol. 468 54:881-941. doi: 10.1146/annurev.micro.54.1.881 (2000).

469 3. Pajuelo, D., Hernández-Cabanyero, C., Sanjuan, E., Lee, C. T., Silva-Hernández, F. X., Hor, 470 L. I., MacKenzie, S. \& Amaro, C. Iron and Fur in the life cycle of the zoonotic pathogen Vibrio 471 vulnificus. Environ. Microbiol. 18(11):4005-4022. doi: 10.1111/1462-2920.13424 (2016).

472 4. Hantke, K. Iron and metal regulation in bacteria. Curr. Opin. Microbiol. 4(2):172-7. doi: 473 10.1016/s1369-5274(00)00184-3 (2001).

5. Litwin, C. M. \& Quackenbush, J. Characterization of a Vibrio vulnificus LysR homolog, 475 HupR, which regulates expression of the haem uptake outer membrane protein, HupA. Microb. 476 Pathog. 31(6):295-307. doi: 10.1006/mpat.2001.0472 (2001).

477 6. Wen, Y., Kim, I. H., Son, J. S., Lee, B. H. \& Kim, K. S. Iron and quorum sensing coordinately 478 regulate the expression of vulnibactin biosynthesis in Vibrio vulnificus. J. Biol. Chem. 287(32): 479 26727-26739. doi: 10.1074/jbc.M112.374165 (2012).

7. Kim, I.H., Wen, Y., Son, J. S., Lee, K. H. \& Kim, K. S. The Fur-iron complex modulates the 481 expression of the quorum-sensing master regulator SmcR to control the expression of virulence 482 factors in Vibrio vulnificus. Infect. Immun. 81(8):2888-98. doi: 10.1128/IAI.00375-13 (2013).

8. Wen, Y., Kim, I. H. \& Kim, K. S. Iron- and quorum-sensing signals converge on small 484 quorum-regulatory RNAs for coordinated regulation of virulence factors in Vibrio vulnificus. J. Biol. Chem. 291(27): 14213-14230. doi: 10.1074/jbc.M116.714063 (2016). 
99. doi: 10.1146/annurev.micro.55.1.165 (2001).

488

489

490

491

492

493

494

495

496

497

498

499

500

501

502

503

504

505

506

507

10. Ng, W. L. \& Bassler, B. L. Bacterial quorum-sensing network architecture. Annu. Rev. Genet. 43:197-222. doi: 10.1146/annurev-genet-102108-134304 (2009).

11. Schuster, M., Sexton, D. J., Diggle, S. P. \& Greenberg, E. P. Acyl-homoserine lactone Quorum sensing: from evolution to application. Annu. Rev. Microbiol. 67:43-63. doi: 10.1146/annurev-micro-092412-155635 (2013).

12. Chen, X., Schauder, S., Potier, N., Van Dorsselaer, A., Pelczer, I., Bassler, B. L. \& Hughson, F. M. Structural identification of a bacterial quorum-sensing signal containing boron. Nature 415(6871):545-9. doi: 10.1038/415545a (2002).

13. Kim, S. Y., Lee, S. E., Kim, Y. R., Kim, C. M., Ryu, P. Y., Choy, H. E., Chung, S. S. \& Rhee, J. H. Regulation of Vibrio vulnificus virulence by the LuxS quorum-sensing system. Mol. Microbiol. 48(6):1647-64. doi: 10.1046/j.1365-2958.2003.03536.x (2003).

14. Park, J. H., Cho, Y. J., Chun, J., Seok, Y. J., Lee, J. K., Kim, K. S., Lee, K. H., Park, S. J. \& Choi, S. H. Complete genome sequence of Vibrio vulnificus MO6-24/O. J. Bacteriol. 193(8):2062-3. doi: 10.1128/JB.00110-11 (2011).

15. Bassler, B. L., Wright, M. \& Silverman, M. R. Multiple signaling systems controlling expression of luminescence in Vibrio harveyi: sequence and function of genes encoding a second sensory pathway. Mol. Microbiol. 13(2):273-86. doi: 10.1111/j.13652958.1994.tb00422.x (1994).

16. Bassler, B. L., Wright, M. \& Silverman, M. R. Sequence and function of LuxO, a negative regulator of luminescence in Vibrio harveyi. Mol. Microbiol. 12(3):403-12. doi: 
17. Freeman, J. A. \& Bassler, B. L. A genetic analysis of the function of LuxO, a two510 component response regulator involved in quorum sensing in Vibrio harveyi. Mol. Microbiol. 31(2):665-77. doi: 10.1046/j.1365-2958.1999.01208.x (1999)

18. Freeman, J. A. \& Bassler, B. L. Sequence and function of LuxU: a two-component phosphorelay protein that regulates quorum sensing in Vibrio harveyi. J. Bacteriol. 181(3):899-906. doi: 10.1128/JB.181.3.899-906.1999 (1999).

19. Milton, D. L. Quorum sensing in Vibrios: complexity for diversification. Int. J. Med. Microbiol. 296(2-3):61-71. doi: 10.1016/j.ijmm.2006.01.044 (2006).

20. Wyman, C., Rombel, I., North, A. K., Bustamante, C. \& Kustu, S. Unusual 518 275(5306):1658-61. doi: 10.1126/science.275.5306.1658 (1997). and sigma-54. Mol. Microbiol. 36(4):940-54. doi: 10.1046/j.1365-2958.2000.01913.x 522 (2000).

22. Roh, J. B., Lee, M. A., Lee, H. J., Kim, S. M., Cho, Y., Kim, Y. J., Seok, Y. J., Park, S.

J. \& Lee, K. H. Transcriptional regulatory cascade for elastase production in Vibrio vulnificus: LuxO activates luxT expression and LuxT represses smcR expression. J. Biol. Chem. 281(46):34775-84. doi: 10.1074/jbc.M607844200 (2006). 
spp. J. Bacteriol. 188(6):2214-21. doi: 10.1128/JB.188.6.2214-2221.2006 (2006).

530

531

532

533

534

535

536

537

538

539

540

541

542

543

544

545

546

547

548

549

24. Kim, I. H., Kim, S. Y., Park, N. Y., Wen, Y., Lee, K. W., Yoon, S. Y., Jie, H., Lee, K. H. \& Kim, K. S. Cyclo-(L-Phe-L-Pro), a quorum-sensing signal of Vibrio vulnificus, induces expression of hydroperoxidase through a ToxR-LeuO-HU-RpoS signaling pathway to confer resistance against oxidative stress. Infect. Immun. 86(9):e00932-17. doi: 10.1128/IAI.0093217 (2018).

25. Park, N. Y., Kim, I. H., Wen, Y., Lee, K. W., Lee, S., Kim, J. A., Jung, K. H., Lee, K. H. \& Kim, K. S. Multi-factor regulation of the master modulator LeuO for the cyclic-(Phe-Pro) signaling pathway in Vibrio vulnificus. Sci. Rep. 9(1):20135. doi: 10.1038/s41598-019-56855$4(2019)$

26. Massé, E. \& Gottesman, S. A small RNA regulates the expression of genes involved in iron metabolism in Escherichia coli. Proc. Natl. Acad. Sci. USA 99(7):4620-5. doi: 10.1073/pnas.032066599 (2002).

27. Mey, A. R., Craig, S. A. \& Payne, S. M. Characterization of Vibrio cholerae RyhB: the RyhB regulon and role of $r y h B$ in biofilm formation. Infect. Immun. 73(9): 5706-5719. doi: 10.1128/IAI.73.9.5706-5719 (2005).

28. Davis, B. M., Quinones, M., Pratt, J., Ding, Y. \& Waldor, M. K. Characterization of the small untranslated RNA RyhB and its regulon in Vibrio cholerae. J. Bacteriol. 187(12): 40054014. doi: 10.1128/JB.187.12.4005-4014 (2005).

29. Kim, J. N. \& Kwon, Y. M. Genetic and phenotypic characterization of the RyhB regulon in Salmonella Typhimurium. Microbiol. Res. 168(1):41-9. doi: 10.1016/j.micres.2012.06.007 
30. Deng, Z., Meng, X., Su, S., Liu, Z., Ji, X., Zhang, Y., Zhao, X., Wang, X., Yang, R. \& Han,

Y. Two sRNA RyhB homologs from Yersinia pestis biovar microtus expressed in vivo have differential Hfq-dependent stability. Res. Microbiol. 163(6-7):413-8. doi: 10.1016/j.resmic.2012.05.006 (2012).

31. Gottesman, S. The small RNA regulators of Escherichia coli: Roles and Mechanisms. Annu. Rev. Microbiol. 58:330-28. doi: 10.1146/annurev.micro.58.030603.123841 (2004). RNA RyhB activates the translation of shiA mRNA encoding a permease of shikimate, a compound involved in siderophore synthesis. Mol. Microbiol. 64(5):1260-73. doi: 10.1111/j.1365-2958.2007.05733.x (2007). and iron-responsive decoding. EMBO. J. 26(4):965-75. doi: 10.1038/sj.emboj.7601553 (2007). RNase III control the stability of $\operatorname{sodB}$ mRNA upon translational inhibition by the small regulatory RNA RyhB. Nucleic. Acids. Res. 33(5):1678-89. doi: 10.1093/nar/gki313 (2005). and its mRNA targets in Escherichia coli. Genes. Dev. 17(19): 2374-2383. doi: 10.1101/gad.1127103 (2003). determines access for small RNA regulator. EMBO. J. 23(2):396-405. doi: 
572 37. Gottesman, S. \& Storz, G. Bacterial small RNA regulators: versatile roles and rapidly 573 evolving variations. Cold. Spring. Harb. Perspect. Biol. 3(12):a003798 (2011).

574 38. Zhang, A., Wassarman, K. M., Rosenow, C., Tjaden, B. C., Storz, G. \& Gottesman, S. 575 Global analysis of small RNA and mRNA targets of Hfq. Mol. Microbiol. 50(4):1111-24. doi: $576 \quad 10.1046 / j .1365-2958.2003 .03734 . x(2003)$.

577 39. McCord, J. M. Iron, free radicals, and oxidative injury. J. Nutr. 134(11):3171S-3172S. doi: 578 10.1093/jn/134.11.3171S (2004).

40. Calderwood, S. B. \& Mekalanos, J. J. Iron regulation of Shiga-like toxin expression in 580 Escherichia coli is mediated by the fur locus. J. Bacteriol. 169(10):4759-64. doi: 581 10.1128/jb.169.10.4759-4764.1987 (1987).

41. Troxell, B. \& Hassan, H. M. Transcriptional regulation by Ferric Uptake Regulator (Fur) in pathogenic bacteria. Front. Cell. Infect. Microbiol. 3:59. doi: 10.3389/fcimb.2013.00059 584 (2013).

42. Lee, D. H., Jeong, H. S., Jeong, H. G., Kim, K. M., Kim, H. \& Choi, S. H. A consensus 586 sequence for binding of SmcR, a Vibrio vulnificus LuxR homolog, and genome-wide 587 identification of the SmcR regulon. J. Biol. Chem. 283(35):23610-8. doi: 588 10.1074/jbc.M801480200 (2008). in the quorum-sensing bacterium Vibrio harveyi. J. Bacteriol. 179(12):4043-5. doi: 10.1128/jb.179.12.4043-4045.1997 (1997). 
592

593

594

595

596

597

598

599

600

601

602

603

604

605

606

607

608

609

610

611

612

44. Jeong, H. S., Lee, M. H., Lee, K. H., Park, S. J. \& Choi, S. H. SmcR and cyclic AMP receptor protein coactivate Vibrio vulnificus vvpE encoding elastase through the RpoSdependent promoter in a synergistic manner. J. Biol. Chem. 278(46):45072-81. doi: 10.1074/jbc.M308184200 (2003).

45. Wassarman, K. M. Small RNAs in bacteria: diverse regulators of gene expression in response to environmental changes. Cell 109(2):141-4. doi: 10.1016/s0092-8674(02)00717-1 (2002).

46. Møller, T., Franch, T., Højrup, P., Keene, D. R., Bächinger, H. P., Brennan, R. G. \& ValentinHansen, P. Hfq: a bacterial Sm-like protein that mediates RNA-RNA interaction. Mol. Cell. 9(1):23-30. doi: 10.1016/s1097-2765(01)00436-1 (2002).

47. Mathews, D. H., Sabina, J., Zuker, M. \& Turner, D. H. Expanded sequence dependence of thermodynamic parameters improves prediction of RNA secondary structure. J. Mol. Biol. 288(5):911-40. doi: 10.1006/jmbi.1999.2700 (1999).

48. Wright, A. C., Simpson, L. M. \& Oliver, J. D. Role of iron in the pathogenesis of Vibrio vulnificus infections. Infect. Immun. 34(2):503-7. doi: 10.1128/iai.34.2.503-507.1981 (1981).

49. Patel, M., Isaäcson, M. \& Gouws, E. Effect of iron and $\mathrm{pH}$ on the survival of Vibrio cholerae in water. Trans. R. Soc. Trop. Med. Hyg. 89:175-177. http://dx.doi.org/10.1016/00359203(95)90484-0 (1995).

50. Cassat, J. E., Skaar, E. P. Iron in infection and immunity. Cell. Host. Microbe. 13(5):509519. doi: 10.1016/j.chom.2013.04.010 (2013). 
614 S., Oh, T. K., Lee, C. H. \& Lee, J. K. The molecular structure and catalytic mechanism of a 615 quorum-quenching N-acyl-L-homoserine lactone hydrolase. Proc. Natl. Acad. Sci. USA 616 102(49):17606-11. doi: 10.1073/pnas.0504996102 (2005).

617 52. Dong, Y. H., Wang, L. H., Xu, J. L., Zhang, H. B., Zhang, X. F. \& Zhang, L. H. Quenching 618 quorum-sensing-dependent bacterial infection by an $\mathrm{N}$-acyl homoserine lactonase. Nature 619 411(6839):813-7. doi: 10.1038/35081101 (2001).

620

621

622

623

624

625

626

627

628

629

630

631

632

633

53. Svenningsen, S. L., Waters, C. M. \& Bassler, B. L. A negative feedback loop involving small RNAs accelerates Vibrio cholerae's transition out of quorum-sensing mode. Genes. Dev. 22(2):226-38. doi: 10.1101/gad.1629908 (2008).

54. Tu, K. C., Waters, C. M., Svenningsen, S. L. \& Bassler, B. L. A small-RNA-mediated negative feedback loop controls quorum-sensing dynamics in Vibrio harveyi. Mol. Microbiol. 70(4):896-907. doi: 10.1111/j.1365-2958.2008.06452.x (2010).

55. Greenberg, E. P., Hastings, J. W. \& Ulitzer, S. Induction of luciferase synthesis in Beneckea harveyi by other marine bacteria. Arch. Microbiol. 120: 87-91 (1979).

56. Milton, D. L., O'Toole, R., Horstedt, P. \& Wolf-Watz, H. Flagellin A is essential for the virulence of Vibrio anguillarum. J. Bacteriol. 178(5):1310-9. doi: 10.1128/jb.178.5.13101319.1996 (1996).

57. Kim, I. H., Kim, I. J., Wen, Y., Park, N. Y., Park, J.Y., Lee, K. W., Koh, A., Lee, J. H., Koo, S. H. \& Kim, K. S. Vibrio vulnificus secretes an insulin-degrading enzyme that promotes bacterial proliferation in vivo. J. Biol. Chem. 290(30):18708-20. doi: 


\section{Acknowledgement}

637 This work was supported by grant from the National Research Foundation, Republic of Korea 638 (NRF-2019R1A2C2084282).

639

\section{Author Contribution Statement}

$641 \mathrm{KL}, \mathrm{YW}$, and NP performed the experiments. KL, YW, and KK were involved in the conception 642 and design of the study. KL and KK were involved in drafting the manuscript.

\section{Competing Interests}

645 We declare that we have no conflicts of interests.

\section{FIGURE LEGENDS}

649 Figure 1. Identification of the transcription start site of RyhB and evidence that Hfq 650 stabilizes the transcript.

651 Hfq stabilizes RyhB RNA. V. vulnificus MO6 and $h f q$ mutant ( $\Delta h f q$ ) were cultured in LB broth 페이지 30 / 36 
for 3 hours, at which point $200 \mu \mathrm{M}$ of 2, 2'-dipyridyl was added and incubation continued for another hour. Samples were then treated with $250 \mathrm{~g} / \mathrm{ml}$ of rifampicin and collected at time intervals for RNA quantification. All primer extensions were performed using primer RyhBPE (Table S3).

\section{Figure 2. Expression of RyhB is dependent on Fur and iron concentrations.}

(a) Primer extensions were carried out to assess the level of RyhB expression with and without Fur and iron. Wild type MO6 and a fur mutant were cultured in LB supplemented with either $25 \mu \mathrm{M}$ of $\mathrm{FeSO}_{4}$ or $200 \mu \mathrm{M} 2,2^{\prime}$-dipyridyl. After RNA extraction, the RyhB transcript was quantified by primer extension using primer RyhB-PE (Table S2). Sequencing ladders generated by the same primer are included. (b) Gel shift assay of a ${ }^{32} \mathrm{P}$-labeled DNA fragment of the region upstream of $r y h B$ with increasing amounts of Fur in the presence of the divalent cation $\mathrm{MnSO}_{4}$. Lanes 1 through 5 are Fur concentrations of 0, 50, 100, 200, and $300 \mathrm{nM}$, respectively. Lanes 6 and 7 include $300 \mathrm{nM}$ of Fur incubated with probe in the presence of either $260 \mathrm{ng}$ or $720 \mathrm{ng}$ of non-labeled probe, respectively. (c) Gel shift assay of ${ }^{32} \mathrm{P}$-labeled $r y h B$ probe with increasing amounts of Fur in the presence of 10 mM EDTA. Lanes 1 through 5 are Fur concentration of $0,50,100,200$, and $300 \mathrm{nM}$, respectively.

\section{Figure 3. The quorum sensing master regulator SmcR represses the expression of $r y h B$.}

Quantitative analysis of $r y h B$ transcription levels using $l u x A B$ as reporter genes. Luminescence activity representing the level of $r y h B$ transcription was compared at early stationary phase of growth $\left(\mathrm{A}_{600} \cong 1.0\right)$ for $V$. vulnificus $\mathrm{MO6}-24 / \mathrm{O}, \Delta l u x O, \Delta s m c R$, and $\Delta l u x O \Delta s m c R$ harboring 
pHK-ryhB. Relative light units (RLU) were normalized to cell density (luminescence/A 600 ). Values are averages from three independent experiments, and error bars denote standard deviations. The $p$-values for comparison with MO6-24/O are indicated (Student's $t$-test; *, $0.005 \leq P<0.05)$.

\section{Figure 4. SmcR binds directly to the upstream region of $r y h B$.}

(a) Gel mobility shift assay of purified SmcR binding to a DNA fragment of the upstream region of $r y h B$. Lanes 1 to 4 represent $20 \mathrm{ng}$ of ${ }^{32} \mathrm{P}$-labeled $r y h B$ probe incubated with $0 \mathrm{nM}$, $62.5 \mathrm{nM}, 125 \mathrm{nM}$, and $250 \mathrm{nM}$ of SmcR, respectively. Lanes 5 and 6 represent $20 \mathrm{ng}$ of the labeled $r y h B$ probe incubated with $250 \mathrm{nM}$ of SmcR with the addition of either $260 \mathrm{ng}$ or 780 ng of unlabeled probe as a competitor, respectively. (b) DNase I footprinting to identify the SmcR binding site in the region upstream of $r y h B .{ }^{32} \mathrm{P}$-labeled $r y h B$ probe $(100 \mathrm{ng})$ with SmcR at $0,62.5,125,250,500$ and $1000 \mathrm{nM}$ is included in lanes 1 to 5 , respectively. Sequencing ladders were included for comparison.

\section{Figure 5. RyhB promotes AI-2 production and vvpE expression.}

(a) Effects of RyhB on AI-2 production were measured using the AI-2 indicator $V$. harveyi strain BB170. V. vulnificus strains wild type MO6-24/O, $\Delta r y h B, \Delta r y h B$ with a $r y h B$ clone $(\Delta r y h B$ pBBR12-ryhB), $\Delta h f q, \Delta h f q$ with a $h f q$ clone $(\Delta h f q$ pBBR12- $h f q)$, and $\Delta l u x S$ were cultured in $\mathrm{AB}$ minimal medium and supernatants were collected to measure AI-2 production. (b) Effects of RyhB on the expression of $v v p E$. $v v p E$ expression was measured using a transcriptional 
695

696

697

698

699

700

701

702

703

704

705

706

707

708

709

710

711

712

713

714

715

716

fusion (pHK-vvpE) in either $V$. vulnificus wild type or the $\Delta r y h B$ mutant. $V$. vulnificus was cultured in LBS medium supplemented with $100 \mu \mathrm{M}$ of 2, 2'-dipyridyl to $\mathrm{A}_{600}$ of about 0.1 . Samples were collected at late stationary phase $\left(\mathrm{A}_{600} \cong 2.5\right)$ and both cell density and luminescence were measured. Relative light units (RLU) were normalized to cell density (luminescence/ $\left.\mathrm{A}_{600}\right)$. Values are an average of three independent experiments and error bars denote the standard deviations. The $p$-values for comparison with MO6-24/O (without iron) are indicated (Student's $t$-test; $*, 0.005 \leq P<0.05 ; * *, P<0.005$ ).

\section{Figure 6. Effects of RyhB on the stability of LuxS mRNA.}

(a) A comparison of LuxS mRNA levels in wild type and $\Delta r y h B . V$. vulnificus MO6-24/O and $r y h B$ mutant $(\Delta r y h B)$ were cultured in LB broth for 3 hours, at which point $200 \mu \mathrm{M}$ of 2, 2'dipyridyl was added and cultures continued incubating for another 30 minutes. Cultures were then treated with $250 \mu \mathrm{g} / \mathrm{ml}$ rifampicin and samples were collected for RNA purification at 0 , 10, 20, 30, 40, and 60 minutes. 16S rRNA was used as a reference for LuxS mRNA quantification. Primer extension was performed using primer LuxS-PE+116 (Table S2). (b) Quantification of luxS mRNA levels. Band intensities from the gel shown in Figure 6A were measured with a Multi Gauge V3.0 (Fujifilm, Tokyo, Japan). (c) Effects of RyhB and Hfq on the stability of LuxS mRNA. The relative levels of luxS mRNA following rifampicin treatment were measured using qRT-PCR. $V$. vulnificus MO6, $r y h B$ mutant $(\Delta r y h B), h f q$ mutant $(\Delta h f q)$ or $r y h B, h f q$ double mutant $(\Delta r y h B \Delta h f q)$ were cultured in AB broth for 5 hours until reaching an $\mathrm{A}_{600}$ of 0.3 . Samples were then collected at $0,2,4,6$, and 8 minutes after the addition of rifampicin $(500 \mu \mathrm{g} / \mathrm{ml})$, and RNA was quantified by qRT-PCR. 
718 Figure 7. Effects of Hfq on interactions between RyhB and LuxS mRNA.

719 (a) Gel shift assay comparing the binding of RyhB to LuxS mRNA with and without Hfq. The 720 LuxS 5'-UTR was transcribed and labeled with ${ }^{32}$ P-UTP using T7 RNA polymerase. Increasing concentrations of purified RyhB were incubated with LuxS 5'-UTR with or without the addition of $1 \mu \mathrm{M}$ of Hfq. Lanes 1 to 4 include RyhB concentrations of $0,100,200$, and $400 \mathrm{nM}$ without Hfq, respectively. Lanes 5 to 8 include RyhB concentrations of $0,100,200$, and $400 \mathrm{nM}$ with Hfq, respectively. The probes bound by RyhB are indicated with arrows. (b) Effects of Hfq on the stability of RyhB mRNA. Relative RyhB mRNA levels following rifampicin treatment were measured using qRT-PCR. $V$. vulnificus MO6 or the $h f q$ mutant $(\Delta h f q)$ were cultured in AB broth for 5 hours until reaching an $A_{600}$ of 0.3 . Samples were then collected at $0,2,4,6$, and 8 minutes after treatment with $500 \mu \mathrm{g} / \mathrm{ml}$ rifampicin for RNA purification and qRT-PCR analysis.

Figure 8. Predicted secondary structure of the $5^{\prime}$ end of LuxS mRNA and potential hybridization sites between RyhB and LuxS 5'-UTR mRNA.

732

(a) The secondary structure of the $5^{\prime}$ end of LuxS mRNA was predicted using mFold software. The nucleotides that potentially form stem structures are shaded in blue (SL1) or red (SL2) and the start codon is noted. (b) Possible regions where RyhB and the LuxS 5'-UTR may hybridize. Three possible regions of hybridization (HR1, HR2, and HR3) are indicated and the potential stem structures from Figure 8A are noted with the corresponding colored dots and arrows. 
(a) Primer extension assays to determine the hybridization sites between $r y h B$ RNA and luxS RNA. Lanes 1 to 4 include Hfq at 0, 125, 250, and $500 \mathrm{nM}$, respectively. Lanes 5 to 9 include LuxS at 100, 200, 400, 600, and $800 \mathrm{nM}$ incubated with $1 \mu \mathrm{M}$ Hfq, respectively. The three distinct bands that represent higher intensities in the presence of Hfq are indicated. Base positions are numbered relative to the transcription start site of RyhB. (b) A model of base pairings between LuxS 5'-UTR and RyhB. Hybridization regions (HR) 1, 2, and 3 are highlighted in yellow. Loops 1 and 2 of RyhB, which form during the hybridization also are indicated. The region labeled HR0 is the site that was mutagenized to generate HR0m as a negative control.

Figure 10. Effects of the hybridization between RhyB and LuxS on expression of LuxS

(a) Influence of hybridization regions HR2 and HR3 on the production of AI-2 by LuxS as measured using the $V$. harveyi indicator strain BB170. The following $V$. vulnificus strains were cultured in $\mathrm{AB}$ minimal medium and sampled for the presence of AI-2 in the supernatant: 1 , wild type (MO6 pBBR12); 2, ryhB deletion mutant ( $\triangle r y h B$ pBBR12); 3, $\Delta r y h B$ with a wild type $r y h B$ clone $(\Delta r y h B$ pBBR12-ryhB); $4, \Delta r y h B$ with a mutated region HR2 clone $(\Delta r y h B$ pBBR12-ryhB HR2m); 5, $\Delta r y h B$ with mutated region HR3 clone ( $\Delta r y h B$ pBBR12-ryhB HR3m); 6, $\Delta r y h B$ carrying a clone with both HR2 and HR3 mutated ( $\triangle r y h B$ pBBR12-ryhB HR2 \&3m); $7, \Delta r y h B$ with a clone carrying an unrelated mutated region as a control $(\Delta r y h B$ pBBR12-ryhB HR0m). The presence of AI-2 was measured in RLU, relative light units (luminescence/ $\mathrm{A}_{600}$ ). Values are averages from three independent experiments and error bars 
760 denote the standard deviations. The $p$-values as compared with MO6-24/O pBBR12 are 761 indicated (Student's $t$-test; * $0.005 \leq P<0.05$; ns, no significant). (b) Effects of HR2 and HR3 762 on the production of LuxS as measured by western hybridization. Western blot analysis of LuxS 763 levels in whole cell lysates was measured. As a control, insulinase (54) enzyme (SidC), which 764 is not regulated by quorum-sensing, was included. (c) The relative mRNA levels of luxS were 765 measured using qRT-PCR for each of the strains listed in 10A. Cells were cultured in AB broth 766 for 5 hours until they reached an $\mathrm{A}_{600}$ of 0.3 , at which point samples were collected for RNA 767 purification and qRT-PCR analysis. The $p$-values for comparison with MO6-24/O pBBR12 are 768 indicated (Student's $t$-test; *, $0.005 \leq P<0.05$; ns, no significant). 
Figures
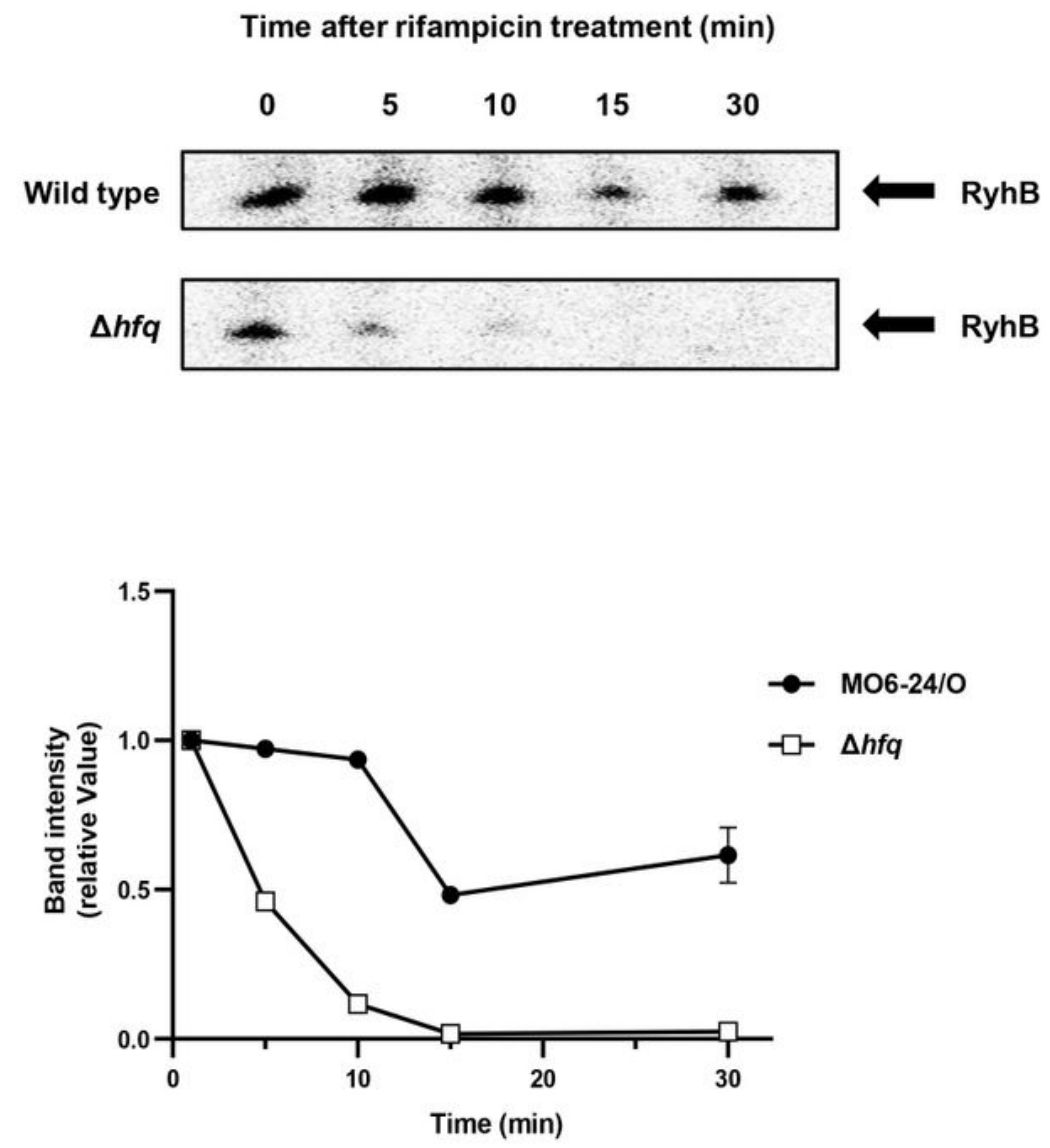

\section{Figure 1}

\section{Figure 1}

Identification of the transcription start site of RyhB and evidence that Hfq stabilizes the transcript. $\mathrm{Hfq}$ stabilizes RyhB RNA. V. vulnificus MO6 and hfq mutant ( $\triangle \mathrm{hfq})$ were cultured in LB broth for 3 hours, at which point $200 \mu \mathrm{M}$ of 2, 2'-dipyridyl was added and incubation continued for another hour. Samples 
were then treated with $250 \mathrm{~g} / \mathrm{ml}$ of rifampicin and collected at time intervals for RNA quantification. All primer extensions were performed using primer RyhB $₫ 655$ PE (Table S3

a

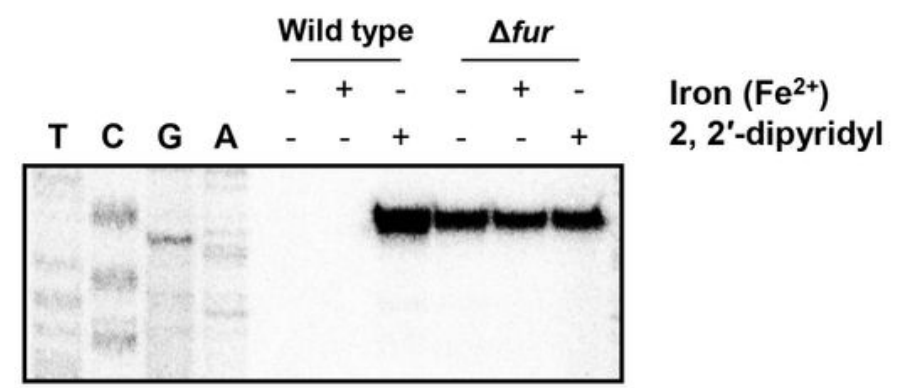

b
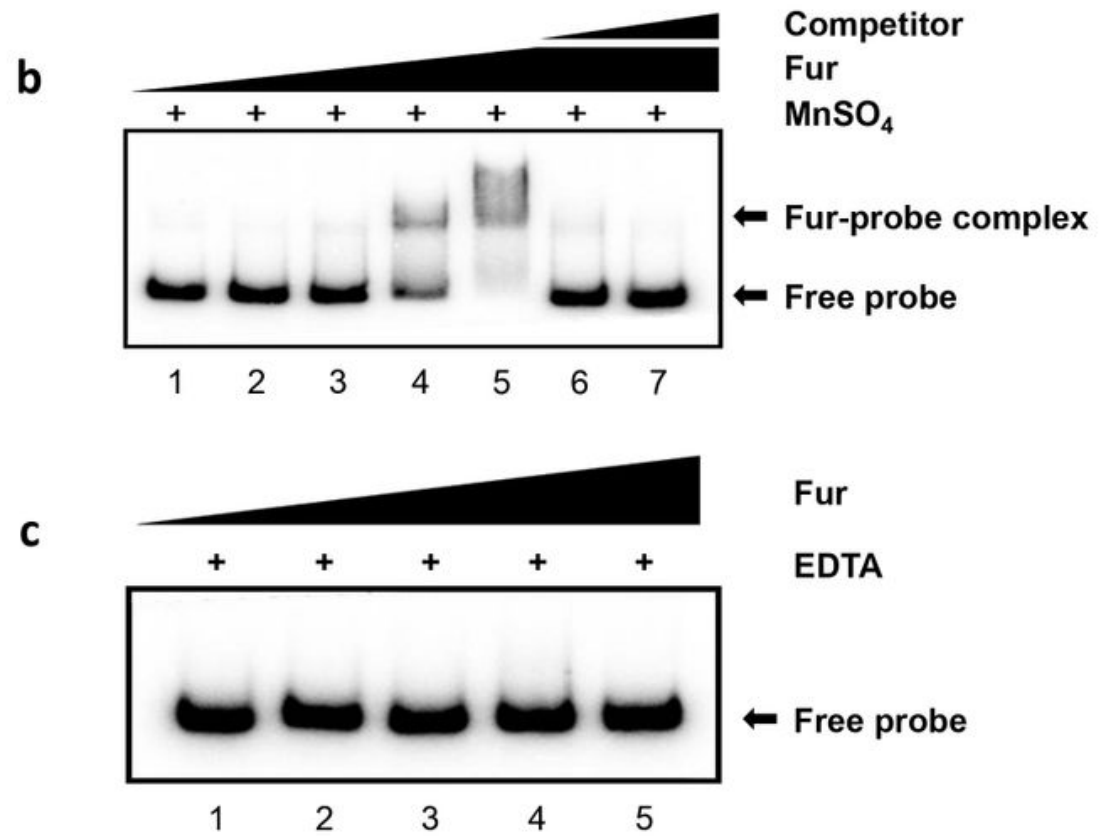

Figure 2

Figure 2

Expression of RyhB is dependent on Fur and iron concentrations. (a) Primer extensions were carried out to assess the level of RyhB expression with and without Fur and iron. Wild type M06 and a fur mutant were cultured in LB supplemented with either $25 \mu \mathrm{M}$ of FeSO4 or $200 \mu \mathrm{M}$ 2, 2『-dipyridyl. After RNA 
extraction, the RyhB transcript was quantified by primer extension using primer RyhB-PE (Table S2).

Sequencing ladders generated by the same primer are included. (b) Gel shift assay of a 32 P-labeled DNA fragment of the region upstream of ryhB with increasing amounts of Fur in the presence of the divalent cation MnSO4. Lanes 1 through 5 are Fur concentrations of $0,50,100,200$, and 300 nM, respectively. Lanes 6 and 7 include $300 \mathrm{nM}$ of Fur incubated with probe in the presence of either $260 \mathrm{ng}$ or $720 \mathrm{ng}$ of non-labeled probe, respectively. (c) Gel shift assay of 32 P-labeled ryhB probe with increasing amounts of Fur in the presence of $10 \mathrm{mM}$ EDTA. Lanes 1 through 5 are Fur concentration of 0, 50, 100, 200, and 300 $\mathrm{nM}$, respectively. 
ryhB::luxAB

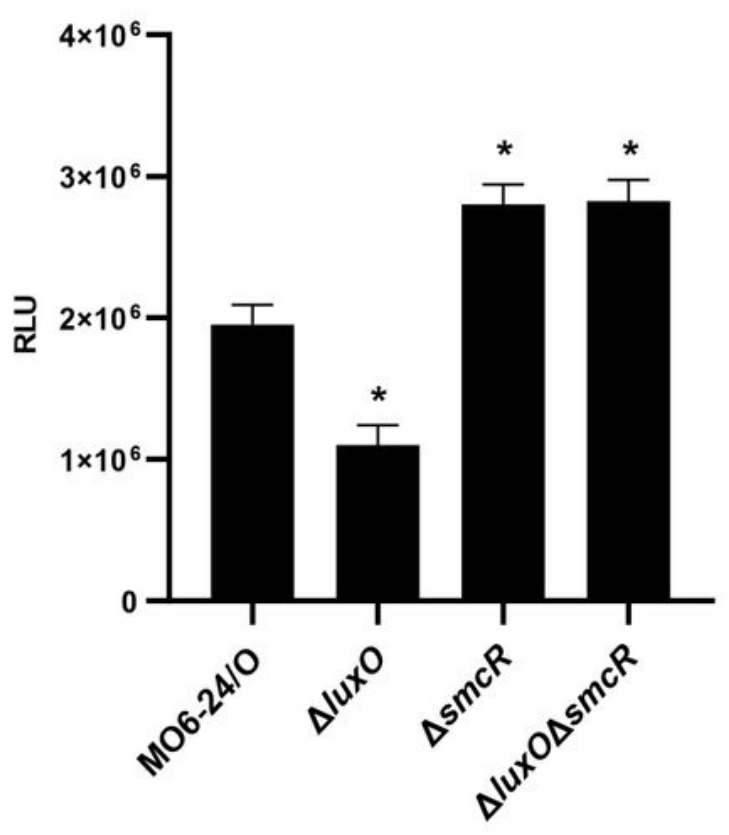

\section{Figure 3}

\section{Figure 3}

The quorum sensing master regulator SmcR represses the expression of ryhB. Quantitative analysis of ryhB transcription levels using luxAB as reporter genes. Luminescence activity representing the level of ryhB transcription was compared at early stationary phase of growth (A600 $\otimes 1.0$ ) for V. vulnificus MO624/O, $\Delta$ luxO, $\Delta s m c R$, and $\Delta$ luxO $\Delta s m c R$ harboring pHK-ryhB. Relative light units (RLU) were normalized to cell density (luminescence/A600). Values are averages from three independent experiments, and error 
bars denote standard deviations. The $\mathrm{p}$-values for comparison with M06-24/0 are indicated (Student's ttest; *, $0.005 \leq \mathrm{P}<0.05)$.

a

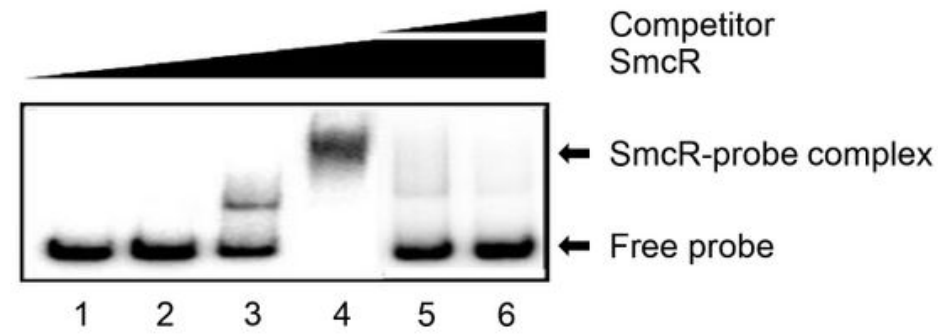

b

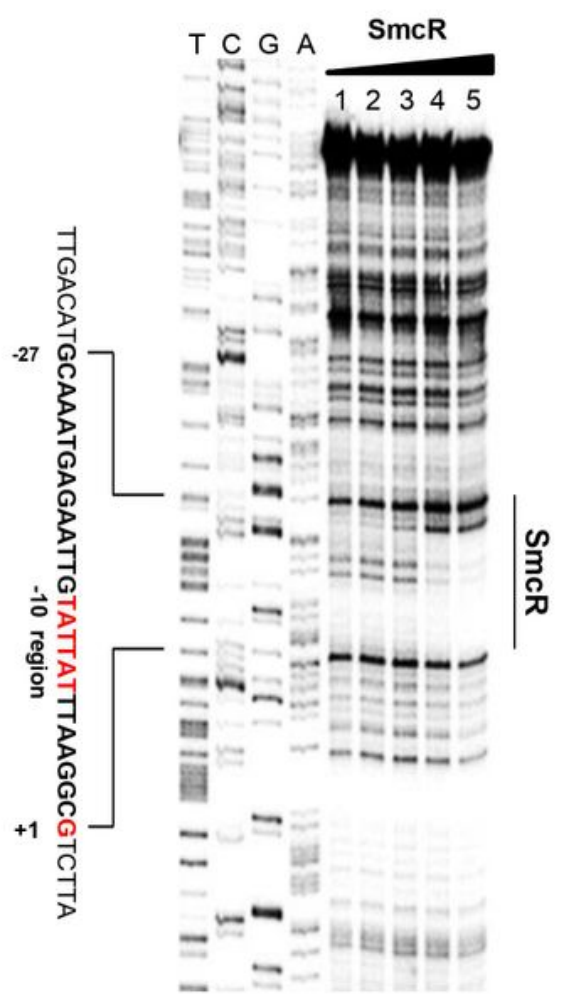

\section{Figure 4}

\section{Figure 4}

SmcR binds directly to the upstream region of ryhB. (a) Gel mobility shift assay of purified SmcR binding to a DNA fragment of the upstream region of ryhB. Lanes 1 to 4 represent $20 \mathrm{ng}$ of 32 P-labeled ryhB probe incubated with $0 \mathrm{nM}, 62.5 \mathrm{nM}, 125 \mathrm{nM}$, and $250 \mathrm{nM}$ of SmcR, respectively. Lanes 5 and 6 represent 
$20 \mathrm{ng}$ of the labeled ryhB probe incubated with $250 \mathrm{nM}$ of SmcR with the addition of either $260 \mathrm{ng}$ or 780 ng of unlabeled probe as a competitor, respectively. (b) DNase I footprinting to identify the SmcR binding site in the region upstream of ryhB. 32 P-labeled ryhB probe (100 ng) with SmcR at 0, 62.5, 125, 250, 500 and $1000 \mathrm{nM}$ is included in lanes 1 to 5 , respectively. Sequencing ladders were included for comparison.

a

\section{Al-2 production}

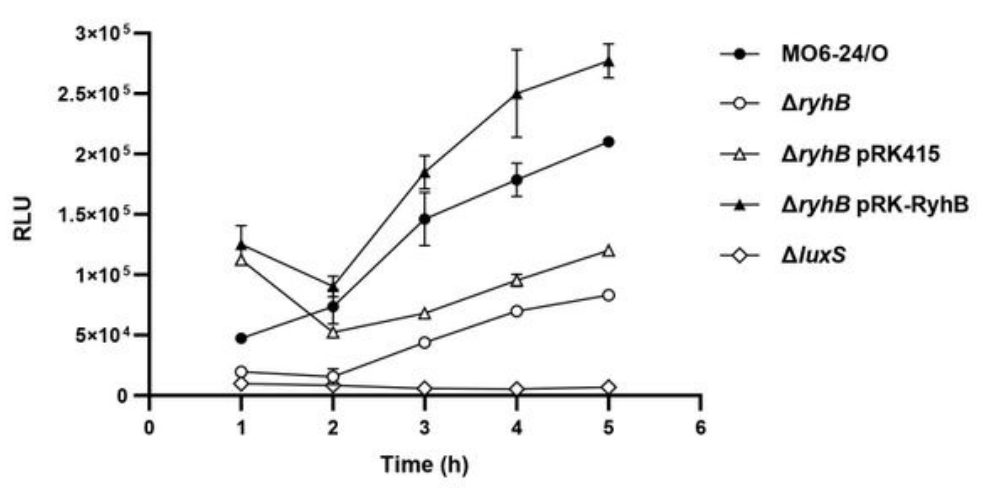

b

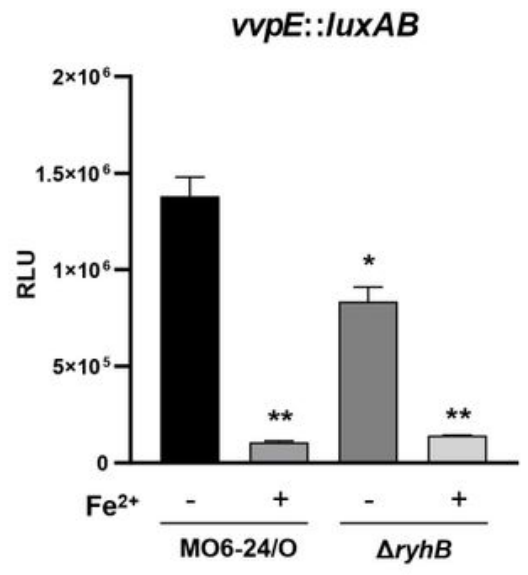

Figure 5

Figure 5 
RyhB promotes Al-2 production and vvpE expression. (a) Effects of RyhB on Al-2 production were measured using the Al-2 indicator V. harveyistrain BB170. V. vulnificus strains wild type M06-24/O, $\Delta$ ryhB, $\Delta$ ryhB with a ryhB clone ( $\Delta$ ryhB pBBR12-ryhB), $\Delta$ hfq, $\Delta$ hfq with a hfq clone ( $\Delta$ hfq pBBR12-hfq), and $\Delta$ luxS were cultured in $A B$ minimal medium and supernatants were collected to measure Al-2 production. (b) Effects of RyhB on the expression of vvpE. vvpE expression was measured using a transcriptional 95 fusion (pHK-vvpE) in either V. vulnificus wild type or the $\triangle$ ryhB mutant. V. vulnificus was cultured in LBS medium supplemented with $100 \mu \mathrm{M}$ of 2, 2'-dipyridyl to A600 of about 0.1. Samples were collected at late stationary phase $(A 600 \otimes 2.5)$ and both cell density and luminescence were measured. Relative light units (RLU) were normalized to cell density (luminescence/A600). Values are an average of three independent experiments and error bars denote the standard deviations. The $\mathrm{p}$-values for comparison with M06-24/O (without iron) are indicated (Student's t-test; *, $0.005 \leq \mathrm{P}<0.05 ; * \star, P<0.005$ ). 


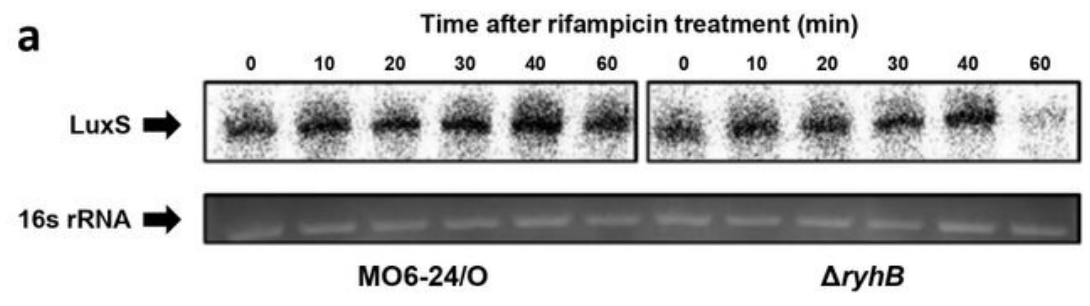

b

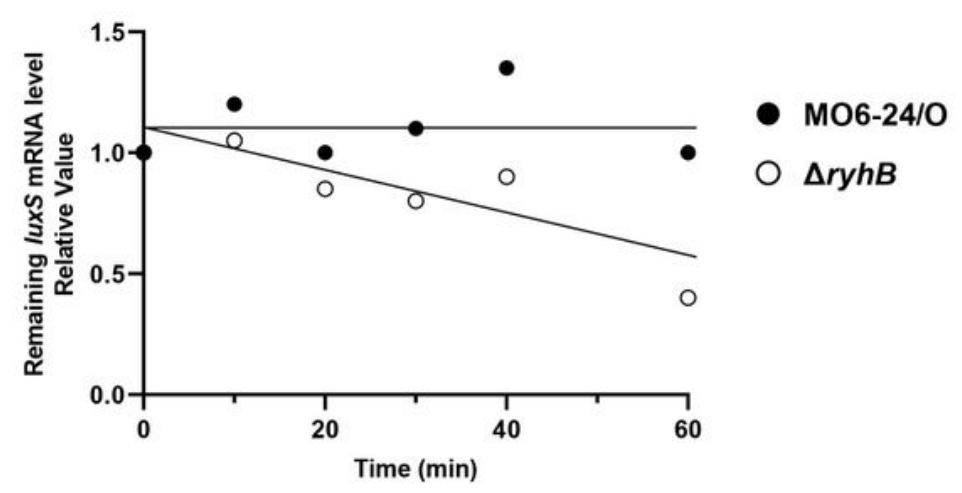

c

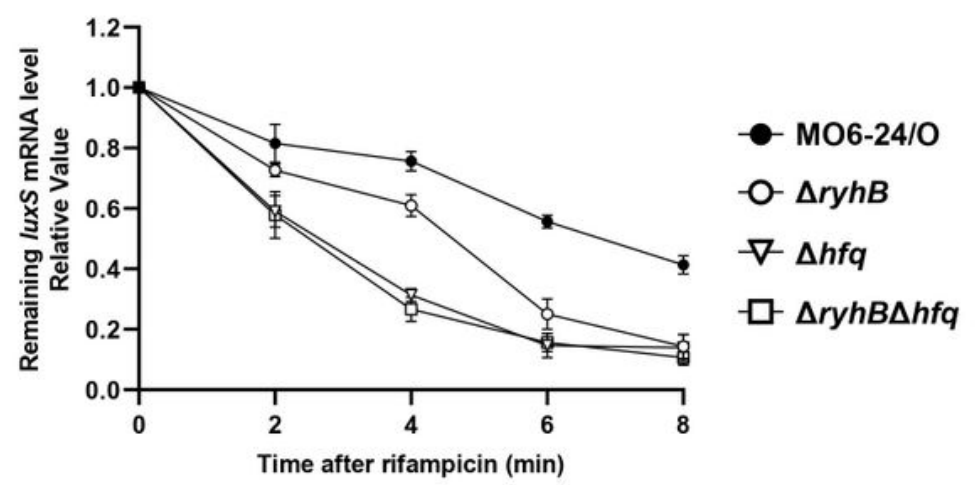

\section{Figure 6}

\section{Figure 6}

Effects of RyhB on the stability of LuxS mRNA. (a) A comparison of LuxS mRNA levels in wild type and $\Delta$ ryhB. V. vulnificus M06-24/O and ryhB mutant $(\triangle \mathrm{ryhB})$ were cultured in LB broth for 3 hours, at which point $200 \mu \mathrm{M}$ of 2, 2'- dipyridyl was added and cultures continued incubating for another 30 minutes. Cultures were then treated with $250 \mu \mathrm{g} / \mathrm{ml}$ rifampicin and samples were collected for RNA purification at $0,10,20,30,40$, and 60 minutes. 16S rRNA was used as a reference for LuxS mRNA quantification. 
Primer extension was performed using primer LuxS-PE+116 (Table S2). (b) Quantification of luxS mRNA levels. Band intensities from the gel shown in Figure 6A were measured with a Multi Gauge V3.0 (Fujifilm, Tokyo, Japan). (c) Effects of RyhB and Hfq on the stability of LuxS mRNA. The relative levels of luxS mRNA following rifampicin treatment were measured using qRT-PCR. V. vulnificus MO6, ryhB mutant $(\Delta r y h B)$, hfq mutant $(\Delta h f q)$ or ryhB, hfq double mutant $(\Delta r y h B \Delta h f)$ were cultured in AB broth for 5 hours until reaching an $A 600$ of 0.3 . Samples were then collected at $0,2,4,6$, and 8 minutes after the addition of rifampicin $(500 \mu \mathrm{g} / \mathrm{ml})$, and RNA was quantified by qRT-PCR.

a

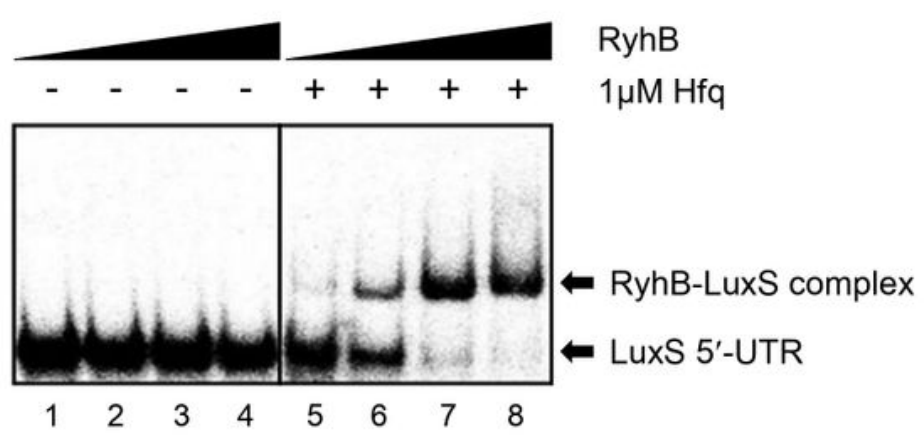

b

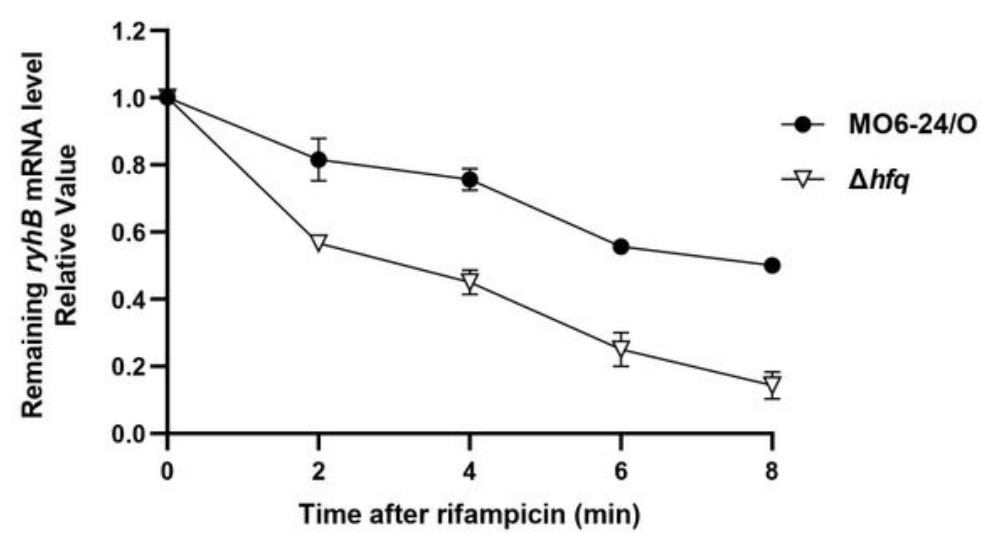

Figure 7 


\section{Figure 7}

Effects of Hfq on interactions between RyhB and LuxS mRNA. (a) Gel shift assay comparing the binding of RyhB to LuxS mRNA with and without Hfq. The LuxS 5'-UTR was transcribed and labeled with $32 \mathrm{P}$ UTP using T7 RNA polymerase. Increasing concentrations of purified RyhB were incubated with LuxS 5'UTR with or without the addition of $1 \mu \mathrm{M}$ of Hfq. Lanes 1 to 4 include RyhB concentrations of $0,100,200$, and $400 \mathrm{nM}$ without Hfq, respectively. Lanes 5 to 8 include RyhB concentrations of 0, 100, 200, and 400 $\mathrm{nM}$ with $\mathrm{Hfq}$, respectively. The probes bound by RyhB are indicated with arrows. (b) Effects of Hfq on the stability of RyhB mRNA. Relative RyhB mRNA levelsfollowing rifampicin treatment were measured using qRT-PCR. V. vulnificus MO6 or the hfq mutant $(\Delta \mathrm{hfq})$ were cultured in AB broth for 5 hours until reaching an $A 600$ of 0.3 . Samples were then collected at $0,2,4,6$, and 8 minutes after treatment with $500 \mu \mathrm{g} / \mathrm{ml}$ rifampicin for RNA purification and qRT-PCR analysis.

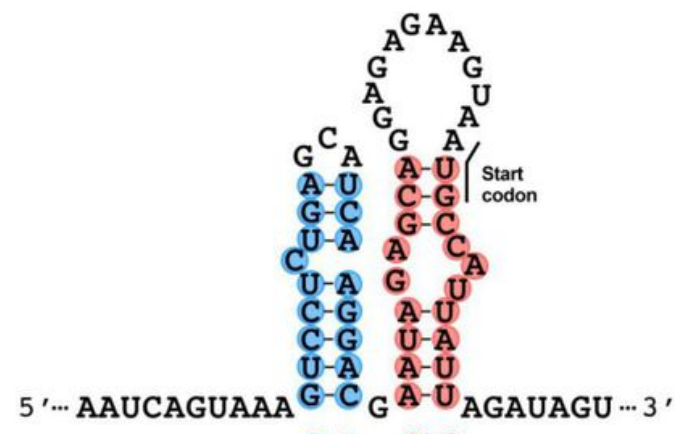

SL $1 \quad$ SL 2

b

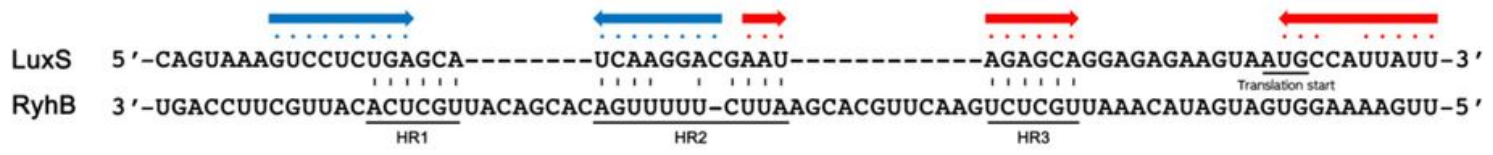

Figure 8

\section{Figure 8}

Predicted secondary structure of the 5, end of LuxS mRNA and potential hybridization sites between RyhB and LuxS 5'-UTR mRNA. (a) The secondary structure of the 5 ' end of LuxS mRNA was predicted using 
mFold software. The nucleotides that potentially form stem structures are shaded in blue (SL1) or red (SL2) and the start codon is noted. (b) Possible regions where RyhB and the LuxS 5囚-UTR may hybridize. Three possible regions of hybridization (HR1, HR2, and HR3) are indicated and the potential stem structures from Figure $8 \mathrm{~A}$ are noted with the corresponding colored dots and arrows.

a

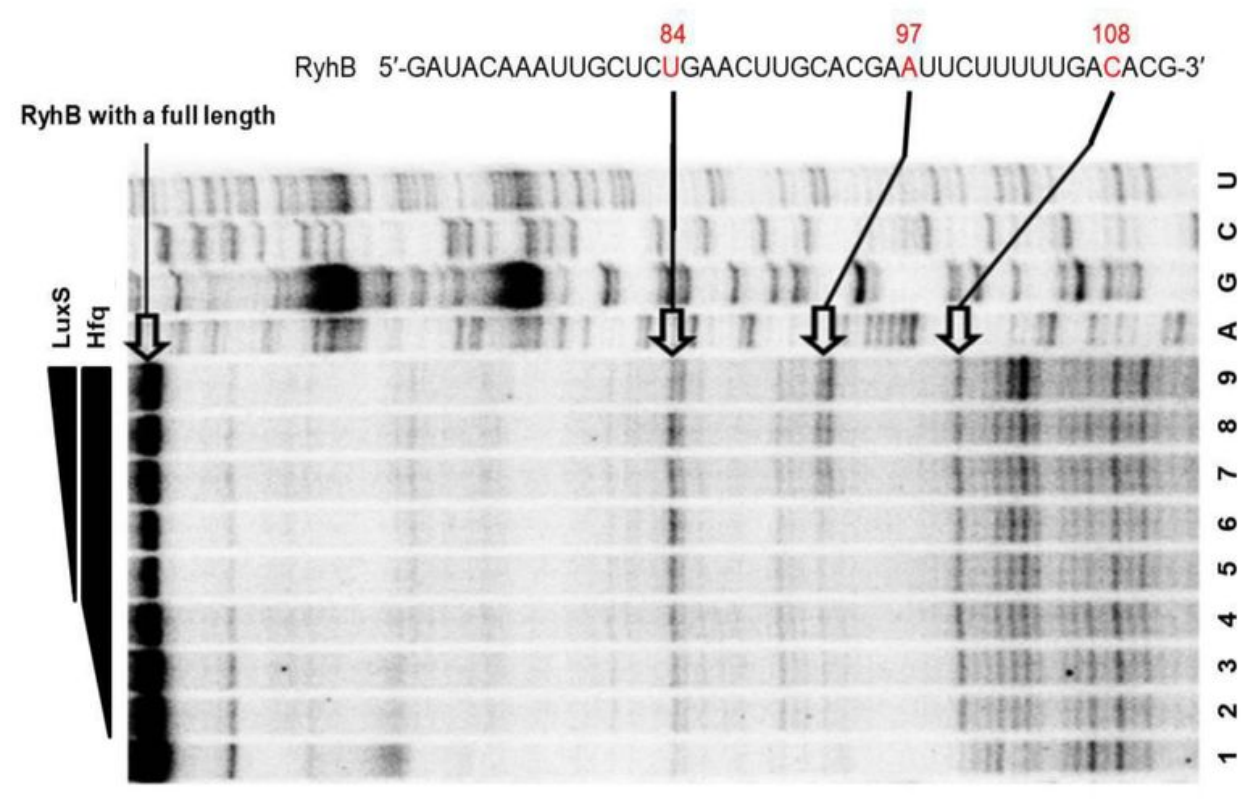

b
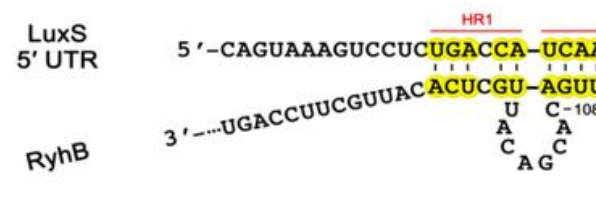

Loop 1

Figure 9

\section{Figure 9}

Identification of regions of RyhB RNA that hybridize with LuxS mRNA. (a) Primer extension assays to determine the hybridization sites between ryhB RNA and luxS RNA. Lanes 1 to 4 include Hfq at 0, 125, 250, and $500 \mathrm{nM}$, respectively. Lanes 5 to 9 include LuxS at 100, 200, 400,600, and $800 \mathrm{nM}$ incubated with $1 \mu \mathrm{M} \mathrm{Hfq}$, respectively. The three distinct bands that represent higher intensities in the presence of $\mathrm{Hfq}$ are indicated. Base positions are numbered relative to the transcription start site of RyhB. (b) A model of base pairings between LuxS 5'-UTR and RyhB. Hybridization regions (HR) 1, 2, and 3 are highlighted in yellow. Loops 1 and 2 of RyhB, which form during the hybridization also are indicated. The region labeled $\mathrm{HRO}$ is the site that was mutagenized to generate HROm as a negative control. 
a

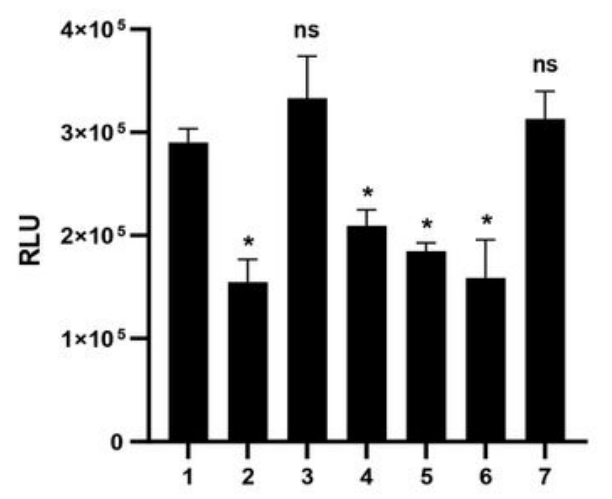

b

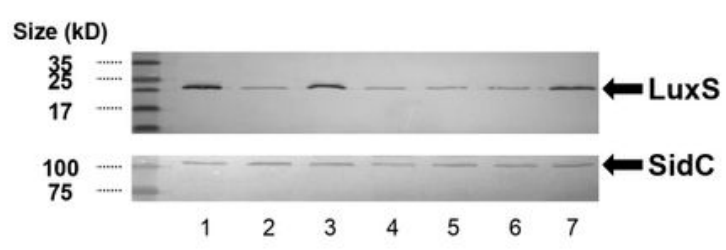

C

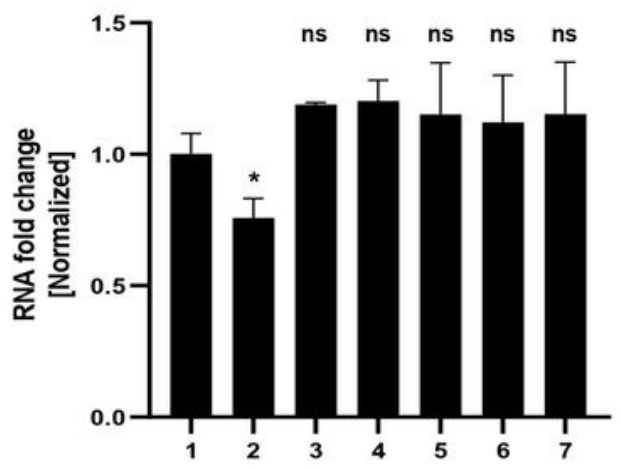

Figure 10

Figure 10

Effects of the hybridization between RhyB and LuxS on expression of LuxS (a) Influence of hybridization regions HR2 and HR3 on the production of Al-2 by LuxS as measured using the V. harveyi indicator strain BB170. The following V. vulnificus strains were cultured in $A B$ minimal medium and sampled for the presence of Al-2 in the supernatant: 1, wild type (M06 pBBR12); 2, ryhB deletion mutant ( $\triangle$ ryhB pBBR12); $3, \Delta$ ryhB with a wild type ryhB clone ( $\triangle$ ryhB pBBR12-ryhB); $4, \Delta$ ryhB with a mutated region HR2 clone 
( $\triangle$ ryhB pBBR12-ryhB HR2m); 5, $\Delta$ ryhB with mutated region HR3 clone ( $\triangle$ ryhB pBBR12-ryhB HR3m); 6 , $\triangle$ ryhB carrying a clone with both HR2 and HR3 mutated ( $\triangle$ ryhB pBBR12-ryhB HR2\&3m); 7, $\Delta$ ryhB with a clone carrying an unrelated mutated region as a control ( $\triangle$ ryhB pBBR12-ryhB HROm). The presence of Al2 was measured in RLU, relative light units (luminescence/A600). Values are averages from three independent experiments and error bars denote the standard deviations. The $p$-values as compared with M06-24/O pBBR12 are indicated (Student's t-test; *, $0.005 \leq \mathrm{P}<0.05$; ns, no significant). (b) Effects of HR2 and HR3 on the production of LuxS as measured by western hybridization. Western blot analysis of LuxS levels in whole cell lysates was measured. As a control, insulinase (54) enzyme (SidC), which is not regulated by quorum-sensing, was included. (c) The relative mRNA levels of luxS were measured using qRT-PCR for each of the strains listed in 10A. Cells were cultured in AB broth for 5 hours until they reached an A600 of 0.3 , at which point samples were collected for RNA purification and qRT-PCR analysis. The $p$ values for comparison with M06-24/O pBBR12 are indicated (Student's t-test; *, $0.005 \leq \mathrm{P}<0.05$; ns, no significant)

\section{Supplementary Files}

This is a list of supplementary files associated with this preprint. Click to download.

- FigureS1.pdf

- Flgures2.pdf

- Flgures4.pdf

- FlgureS5.pdf

- Tables1.pdf

- Tables2.pdf

- Tables3.pdf 